(Aus dem anatomischen Institute zu Breslau.)

\title{
Ueber die Derivate der embryonalen Schlundbogen und Schlundspalten bei Säugethieren.
}

\author{
Von
}

Dr. G. B o r n.

Hierzu Tafel X und XI und 5 HoIzschnitte.

In den letzten Jahren sind zwei umfangreiche Arbeiten von Stieda und Wölfler (Litteraturverz. Nr. IV und V) erschienen, die sich beide mit der Entwicklung der Schilddriise bei Säugethierembryonen beschäftigen. Die Darstellungen, die diese Autoren von der Entwicklung der Drüse geben, differiren aber nicht nur sehr merklich von dem, was bisher ïber dieses vielbehandelte Thema als feststehend angenommen wurde, sondern widersprechen sich auch in einer Reihe der wesentlichsten Punkte untereinander.

Seit Remak herrscht die Anschauung, dass die Schilddrüse aus einer einfachen, in der Medianebene gelegenen Ausstülpung des Epithels der ventralen Wand der embryonalen Mund-Rachenböhle in der Höhe der Copula der zweiten Kiemenbogen entstehe und zwar ganz nach dem allgemeinen Schema der Drüsenentwicklung. Nur eine Abweichung von dem regulären Bilde findet dabei statt; die epitheliale Anlage schniirt sich von ihrem Mutterboden ab und verliert so den Zusammenhang mit dem Epithel des Darmkanals, wird zur „Drüse ohne Austührungsgang". - Die genannten Beobachter statuiren dem gegeniiber ïbereinstimmend eine Entwicklung der Schilddrüse aus paarigen, symmetrischen Anlagen an den Seiten der Schlundwand; über den Ort derselben sind sie aber durchaus nicht einig. Stieda sagt (l. c. p.32):,,die Schilddrüse hat ibre erste epitheliale Anlage in einer paarigen Wucherung des Epithels an der Stelle, wo der Rest der epithelialen Auskleidung einer (der letzten oder vorletzten) Kiemenspalte mit dem Rachenepithel zusammenfliesst." 
Wölfler leitet die Drüse von dem Epithel der nach seiner Darstellung sehr eigenthïmlich gestalteten ersten Kiemenspalte her $)$. Ebenso wie die Angaben über den Ort, differiren die specielleren Beschreibungen und Abbildungen der Anlagén selbst so vollkommen, dass es klar ist, dass die genannten Autoren nicht dieselben Gebilde vor Augen gehabt haben können.

1) Hier muss ich gleich eines Irrthums Wölfler's gedeuken, der sich durch seine ganze Darstellung hindurchzieht und der die von mir noch späterhin hervorzuhehende Thatsache illustrirt, dass dem Autor die Orientirung an den von ihm gewählten Sagittalschnitten nicht genügend gelungen ist. Die von ihm in Bezug auf ihre complicirte Form gut beschriebene und durch das Schema Fig. h p. 17 anschaulich illustrirte Schlundspalte ist nicht, wie Wölfler meint, die erste, die tubotympanische, sondern die zweite. Die erste Schlundspalte hat niemals die angegebene Form, wohl aber stimmt die nächstfolgende, die zweite, ganz mit W.'s Beschreibung überein.

Es lässt sich die Unrichtigkeit der Bezeichnung Wölfler's auch aus den Figuren des Autors selbst beweisen. Figur 1 auf Tafel I ist ein schematisches Bild zur Darstellung der Topographie der Schilddrüsen-Anlage nach den bisherigen Anschauungen. Zehn Tage alter Kaninchen-Embryo nach Kölliker. Auf diesem Bilde ist die ersteKiemenarterie hinter das Ohrbläschen gezeichnet, demnach wird auch die erste Kiemenspalte hinter demselben gesucht; erste Kiemenarterie und erste Kiemenspalte liegen aber bekanntlich vor dem Labyrinthe, dicht hinter oder auf einem Querschnitte mit demselben folgt dann die zweite Kiemenspalte. Offenbar hat Kölliker's (Nr. 8 p. 253) Fig. 175 dem Schema Wölflers zu Grunde gelegen; dieselbe zeigt aber die Lage der Kiemenspalten ganz richtig! - Auch in einer ganzen Reihe wesentlicher Punkte erscheint die Copie dem Original gegenüber nicht zum Vortheil verändert. Bei Kölliker tritt die Aortenwurzel am hinteren Rande der vereinigten Unterkieferfortsätze an den Mundhöhlenboden und verläuft demselben angeheftet nach hinten; - bei Wölfler tritt dieselbe in den dritten Kiemenbogen ein und zieht von da nach vorn, so dass der erste Aortenbogen als der letzte Zweig erscheint, den dieselbe abgiebt; - das Labyrinthbläschen ist in der Copie viel zu stark dorsalwärts verschoben; die Zahl der Urwirbel zwischen Labyrinth und oberer Extremität ist um 2 vermehrt; - die ganze Zeichnung endlich ist umgedreht und dadurch erscheint die Herzschlinge nach der falschen Seite ausgebogen. - Abgesehen übrigens von der falschen Eintragung der Aortenbogen in das Schema lässt sich der Irrthum Wölf ler's auch aus einer grossen Zahl seiner übrigen Figuren zur Evidenz nachweisen; es fehlen der Schlundspalte, die er als erste bezeichnet, vollkommen die von Anfang an vorhandenen Beziehungen zum Labyrinthbläschen a. s. w. 
Der eklatante Widerspruch, in dem sich die beiden genannten nenesten Autoren sowohl untereinander, als auch mit den übereinstimmenden Resuliaten der gewichtigsten Autoren auf dem Gebiete der Entwickelung:geschichte befinden, veranlasste mich das Thema mit möglichst strengen Methoden, wie sie namentlich $\mathrm{H}$ is inmer und immer wieder formulirt und als nnerlässlich für derartige Untersuchungen betont hat, von Neuem in Angriff zu nehmen. Da es äusserst schwierig ist, die erste selbstverständliche Bedingung einer erfolgreichen Untersuchung, nämlich die Beschaffung einer continuirlichen Serie von Embryonalstadien, für mehrere Säugethierarten in gleicher Vollkommenheit zu erfiullen, so habe ich mich auf die eine Art beschränkt, von der mir ein genügendes Material zu Gebote stand, das war Sus scropha. Dass die bei dieser Art erhaltenen Resultate auf' andere Säugethiere übertragbar sind, ergiebt die Uebereinstimmung einer Reihe von Bildern, die von den Autoren nach andern Arten gezeichnet wurden, mit den mir bekannten Bildern von Embryonen des Schweines.

Die Untersuchung, die zuerst allein auf die Entwicklung der Thyreoidea gerichtet wai, erweiterte sich unter der Hand zu einer Bearbeitung der Schicksale der Schlundbogen und Schlundspalten, sowie der Entwicklung der Gebilde, die zu denselben in Beziehung stehen; es sind dies namentlich Zunge and Thymus. In einem Puncte muss ich die Erwartungen, die der Titel dieser Arbeit erwecken könnte, beschränken: die Umbildung des Unterkieferbogens, sowie der ersten Kiemenspalte, der tubo-tympanischen, habe ich, da dieselben genügend bekannt sind, nicht weiter verfolgt. Dem Ausgangspuncte gemäss haben die epithelialen Gebilde vorzuiglich die Aufmerksamkeit festgehalten; Gefässe, Knorpel n. s. w. sind nur so weit beschrieben, als es unbedingt nöthig war. Es wurden möglichst vollkommene Querschnittserien von bekannter, grleichmässiger Dicke von den Objecten hergestellt und dann mit Hilfe einer vorher von dem Embryo in bestimmter Vergrösserung genommenen Umrisszeichnung eine plastische Reconstruction der interessirenden Theile ausgeführt. In welcher Weise ich diese Reconstructionen seit Jahren übe, ist in meiner Arbeit ,Ueber die Nasenhöhlen und den Thränennasengang der Amphibien" (Morpholog. Jahrbuch II p. 579) nachzulesen; iiber die weitere Ansbildung dieser Methode hoffe ich demnächst in einem besonderen kleinen Aufsatze zu berichten. Uebrigens habe ich auch die von His empfohlenen 
Flächenconstructionen u. s. f. nicht vernachlässigt. Die Schnittrichtung wurde fast ausschliesslich senkrecht auf die Profilcontur zwischen Scheitel- und Nackenbeuge gewählt; es entspricht dies ungefähr der von Stie da benutzten Schnittrichtung. Die Schnittdicke betrug 1/30 oder $1 / 40 \mathrm{~mm}$, für einzelne specielle Zwecke bin ich auf $1 / 50$ oder $1 / 60 \mathrm{~mm}$ herabgègangen. Die Embryonen waren in Müller'scher Flüssigkeit oder concentrirter Pikrinsäure erhärtet und dann in $90 \%$ Alcohol conservirt, wenige hatten ChromsäureBehandlung erfahren.

Zum Färben der ganzen Stücke diente mit Vortheil eine mit Alaun gekochte Cochenillelösung, die aber frisch sein muss, wenn sie gut durchfärben soll, oder die von Ehrlich angegebene saure Hämatoxylinlösung, die ich bei Herrn Professor Heidenhain kennen gelernt hatte.

Als Maasse habe ich die von His (Nr. 1 p. 12 und ganz neuerdings Nr. 2 p. 5) eingeführten benutzt.

Bei jüngeren Embryonen mit starken Krümmungen am Kopf- und Steissende misst dieser Autor die Länge vom Nackenhöcker bis zu dem am meisten vorspringenden Theile der Steisskrümmung und bezeichnet dieses Maass als Nackenlinie. Die His'sche Bezeichnung hat den Mangel, dass dieselbe nur einen Endpunct der Maasslinie angibt; der andere Endpunct ist sehr variabel, bei Embryo a (Nr. I Taf. $8 \propto 3$ ) trifft derselbe den ersten, bei Embryo A Taf. 1 den letzten Lendenwirbel; es bleibt also nichts übrig, als die von His gewählte Bezeichnung zu acceptiren; der Kürze wegen setzte ich dafür N. L. Da meine Arbeit allmählich zu älteren Embryonen ansteigt, bei denen die Nackenbeuge sich ausgleicht, muss später an Stelle der Nackenlinie die grösste Gerade, die zwischen den Endpuncten des natürlich zusammengekrümmten Embryos zu ziehen ist, genommen werden; ich bezeichne dieselbe nach ihren Endpuncten als SteissScheitellinie S. S. - Bei den Uebergangsstadien sind beide Maasse angegeben.

Die gewöhnlichen, zur Orientirung gewählten Bezeichnungen: „vorn, hinten, oben, unten " führen bei unsern Objecten leicht zu Missverständnissen, da selbst, wenn man die Säugethier-Embryonen in die aufrechte Stellung des Mienschen bringt, so dass die Bauchfläche zur vordern wird, die starken Krümmungen am Kopf- und Steissende, die ausserdem während der Entwicklung weehseln, Schwierigkeiten für die Bezeichnung machen. Lässt man die ventrale Rumpffäche nach vorn sehen, so sind in gewissen Stadien ihre Fortsetzungen am Kopf- und Steissende nach oben und unten, ja schliesslich sogar nach hinten gerichtet; in andern Stadien dagegen wieder nach vorn und dergl. mehr. Mir scheint, dass man diesen Schwierigkeiten am besten aus dem Wege geht, wenn man alle Ortsbezeichnungen auf die jeweilige gekrümmte Körperaxe, die ja durch die Basis des Schädels und die Wirbel- 
säule resp. Chorda annähernd gegeben ist, bezieht, und dabei immer das Stück der Axe berücksichtigt, auf dessen Querschnitt die fraglichen Theile gelegen sind.

Medial und lateral sind nicht misszuverstehen. Die auf diese und zur Axe senkrechten Richtungen können an jeder Stelle und an jedem Embryo als dorsal uud ventral bezeichnet werden, man muss nur beriicksichtigen, dass der Ausdruck: „ein Theil liegt dorsal zu einem andern" immer nur in Bezug auf das zu diesem Theil gehörige Axenstück gemeint ist.

Die Richtung nach dem einen Ende der Axe selbst heisst oralwärts, nach dem andern aboralwärts: - der Kürze wegen habe ich dafür meist den Ausdruck vorn und hinten (resp. vorwärts und rückwärts) gebraucht.

Voraussetzung für diese Bezeichnungsweise ist natürlich die Kenntniss der jedesmaligen Axenkrümmung; ich sehe aber nicht ein, wie man ohne diese überhaupt durchkommen kann. Sohwierigkeiten entstehen nur an solchen Stellen, wo die Körperaxe eine sehr plötzliche Richtungsänderung durchmacht, doch scheinen mir dieselben noch erheblich geringer, als diejenigen, die dadurch hervorgerufen werden, dass man sich, wie es häufig geschieht, die Embryonen gestreckt denkt, und dann die beim Erwachsenen gebräuchlichen Bezeichnungen verwendet.

Es sind zu unterscheiden: äussere und innere Kiemenfurchen, in diesen die äusseren und inneren Oeffnungen der Kiemen- oder Schlundspalten, wobei ich bemerken muss, dass ich an Stelle der sogenannten äussern Oeffnung der Schlundspalten, wie schon His (Nr. 3 p. 319-321) jungst sehr richtig hervorgehoben hat, keinen wirklichen Durchbruch finden konnte, sondern nur eine Verschmelzung des Schlundspaltenepithels mit dem Epithel der äussern Kiemenfurche; doch werde ich im Text der Kürze wegen auch von äusseren Schlundspaltenöffnungen reden. Der zwischen den beiden Ueffnungen gelegene, oft recht complicirte Raum stellt die eigentliche Kiementasche oder Spalte dar.

Ein ziemlich vollkommenes Litteraturverzeichniss hat Wölfle r (Nr. 5 p. 52-50) gegeben. Kurze historische Uebersichten der wesentlichsten Anschauungen uber unser Thema findet man in den Einleitungen beider oben genannten Arbeiten; ich lasse daber zuerst meine eigenen Untersuchungen in Zusammenhang folgen und werde an diese nachher eine Vergleichung mit den Angaben Wölfler's, Stieda's und denen anderer Autoren anschliessen.

\section{Embryonen von $7 \mathrm{~mm} \mathrm{~N}$. L.}

Der allgemeinen Entwickelungshöhe nach erreichten die Schweinsembryonen dieser Grösse, die alle einer Tracht entstammten, beinahe die menschlichen Embryonen, die $\mathrm{H}$ is in Nr. I mit A und B bezeichnet hat. Die vordere 
Extremität bildet, wie bei B Fig. 1 Taf. I, eine gegen die Mitte der Basis verdickte, gegen die Ränder zugeschärfte, dreieckige Platte, deren breiter, festsitzender Rand noch kaum merklich eingeschnürt ist. Die hintere Extremität ist relativ weiter in der Ausbildung zurückgeblieben, als bei dem menschlichen Embryo. Auffällig ist die ungemein grosse Ausdehnung des Wolff'schen Körpers im dorso-ventralen Durchmesser und die relativ kleine Leber. - Die Ausbildung der Kiemenbogen und Kiemenspalten steht in der Mitte zwischen der Ausbildungsstufe derselben bei den menschlichen Embryonen (B und $a$ von $\mathrm{His}$ ); der vierte Kiemenbogen ist noch sichtbar, freilich nicht mehr so vollkommen, wie bei «.

Wie Fig. 1 zeigt, springt bei Embryonen dieser Grösse das ganze Schlındbogengebiet gegen die dorsalwärts angrenzende Stammzone etwas wulstig vor, so dass das dorsale Ende, die Wurzel, jedes Kiemenbogens als eine deutliche Erhebung ansetzt. Riickwärts vom vierten Kiemenbogen setzt sich diese Erhebung durch zwei schwache Rinnen ventralwärts gegen das Herz, dorsalwärts gegen die Stammplatten begrenzt, fort und erreicht etwas abgeschwächt die Anlage der vordern Extremität (vergl. dazu bei $\mathrm{His}$ Nr. 3 p. 318 unten). Man zählt 4 Kiemenbogen und zwischen diesen 3 äussere Kiemenfurchen; der Rest der vierten ist in der Furche zwischen dem kleinen wulstigen vierten Kiemenbogen und der Herzerhebung enthalten. Die Kiemenbogen convergiren ventralwärts, sie werden ihrer Ordnungszahl nach kürzer und stehen weiter von der dorsalen Profilkante ab. War der Kopf des Embryos vor der Härtung ein wenig dorsalwärts aufgebogen, so bemerkt man, dass die ersten Kiemenbogen (Unterkieferbogen) einander in der Mittellinie erreichen, ebenso die vordern Ränder der zweiten; die zwischen ihnen gelegene erste Kiemenfurche schneidet aber nicht bis zur ventralen Mittellinie ein. Daraus folgt, dass in der Mittellinie erster und zweiter Kiemenbogen in einer Fläche zusammenhängen. Freilich beginnt gleich unter dem vordern Rande des zweiten Kiemenbogens der Herzansatz und zerlegt diese Fläche in zwei äusserst schmale Substanzstreifen, welche den Herzansatz divergirend umgeben und ventralwärts von den vordern Enden der Kiementurchen die Kiemenbogen miteinander verbinden. Bei normaler Kopfbeuge sieht man davon nichts; die Kiemenfurchen verschwinden dann in der Längsfurche, die zwischen der Herzerhebung und dem Schlundbogengebiete eingegraben erscheint.

Die erste äussere Kiemenfurche ist ungefähr $0,8 \mathrm{~mm}$ lang, ihre Verlängerung bis zur Rückenkante würde ungefähr ebensoviel messen 
und dieselbe in spitzem Winkel schneiden. Der angrenzende Rand des ersten Kiemenbogens ist in der Mitte etwas eingebogen, so dass zwei flache Randwiilste entstehen. Der zweite Kiemenbogen ist am dorsalen Ende ungefähr 0,7, am ventralen (soweit dasselbe in der Profilansicht sichtbar ist) $0,4 \mathrm{~mm}$ breit; am vordern Rande springt er wie Fig. 1 zeigt, mit drei schwachen Erhebungen in die erste Kiemenfurche ein; der hintere Rand ist ziemlich grade. Der dritte Bogen ist an seinem dorsalen Ende ungefähr 0,35, am ventralen $0,2 \mathrm{~mm}$ breit, also in demselben Sinne, aber nicht so stark keilförmig, wie der zweite, während der erste im umgekehrten Sinne keilförmig erscheint. Die dritte Kiemenfurche ist ungefähr $0,3 \mathrm{~mm}$ lang und nach hinten ein wenig convex ausgebogen. Der vierte Kiemenbogen ist als ein kleines, dreieckiges, wulstiges Feld zu sehen, das unter das Flächenniveau der Ungebung eingegraben ist. Die dorsale Begrenzung derselben ist ein scharfer Umschlagsrand, mit dem sich die Wolff'sche Leiste zu demselben absenkt. Dieser Umschlagsrand trifft auf die dritte Kiemenfurche etwas dorsalwärts von der Höhe ihrer Convexität. In Figur 1 fällt der Rest der vierten Kiemenfurche mit der Grenzfurche der Herzerhebung beinabe zusammen; - ist der Kopf aufgebogen, so sieht man deutlicher, dass der schmale ventrale Verbindungsstreifen der Kiemenbogen auch am ventralen Ende der vierten Kiemenfurche vorbei zur Wo If f'schen Leiste zieht.

Die Mund-Rachenhöhle von Embryonen dieser Grösse ist ein in dorso-ventraler Richtung stark abgeplatteter Spalt, der sich aboralwärts in querem Durchmesser allmählich verengt. Die Axe derselben ist der doppelten Beugung des embryonalen Koptes entsprechend zweimal geknickt. Diese Knickungen sind an der längeren dorsalen Wand (der Decke) eine hintere, an der Stelle der äussern Nackenbenge, - der Knickungswinkel beträgt etwas über einen Rechten; im Anfang des hintern (Rumpf-) Schenkels desselben findet die Theilung der Rachenhöble in Oesophagus und Kehlkopf statt - und eine vordere, an der Stelle der äussern Scheitelbeuge; diese letztere heisst die Sattelbeuge, der Sattelwinkel ist ebenfalls ungefähr ein Rechter. Der Boden, die ventrale Wand des Mund-Rachenhöhlenspaltes, ist kürzer als die Decke. Derselbe zeigt eine der hintern Kopfbeuge entsprechende Knickung. Ueber den Scheitel des Sattelwinkels nach vorn reichen aber jetzt nur die Unterkieferbogen hinaus; nur diese liegen dem vordern. Sckenkel des Sattelwinkels der 
Decke, dem Anfang des Sphenoethmoidaltheils des Schädelgrundes gegenüber. Ueber die Veründerungen dieses Verhältnisses werde ich unten im Zusammenhange berichten. Die Länge der Rachenspalte bis zum Kehlkopfe, der als seichter medianer Einschnitt am Boden beginnt, beträgt ca. $1 \mathrm{~mm}$. Entfernt man an einem Embryo dieser Grösse vorsichtig die Decke der Mundrachenböhle, so gibt der uns hier interessirende Boden folgendes Bild, das ich mir in feineren Verhältnissen noch nach dem Modell ergänzt habe. Die etwas nach vorn convergirenden, plump spindelförmigen Unterkieferfortsätze sind an ihrem Vorderrande in der Mittellinie durch einen tiefen Einschnitt getrennt, der sich auch zwischen den wenig convexen Mundflächen derselben, die dem vordern Schenkel des Sattelwinkels, dem Anfang des Sphenoethmoidaltheiles des Schädels, gegeniuber liegen, fortsetzt. Der hintere Abfall dieser Flächen der Unterkieferfortsätze grenzt aber noch an den hintern Schenkel des Sattelwinkels der Decke, so dass die stumpfen Kanten, die in den Sattelwinkel der Decke selbst eingreifen, noch auf die Unterkieferbogen fallen. In der Mitte setzt sich der obenerwähnte Einschnitt bis zur hintern Grenze des Unterkieferbogens und etwas dariber hinaus fort. Die nächstfolgenden, leistenförmtgen Erhebungen beginnen jederseits am Aussenrande des Bodens dicht hinter den Unterkieferbogenfortsätzen, folgen aber nicht dem Verlaufe der letzteren, sondern convergiren nach rückwärts; es sind ziemlich steil stufenförmig erhobene Leisten, die gegen die Mittellinie zugleich etwas aufsteigen. Ein Blick auf das Modell lehrt, dass dieselben in den ventralen Verlängerungen der zweiten Kiemenbogen liegen. Zwischen ihnen und den etwas nach vorn convergirenden Unterkieferfortsätzen bleibt am Boden der Mundhöhle ein dreieckiges oder durch einen schwachen Winkel am Vorderrande viereckiges Feld, eine Art „Schaltstiuck", das vorn durch zwei schwache, hinten durch zwei schärfere Furchen abgesetzt ist. Obgleich in Bezug auf die Einzelheiten und in den Dimensionen schon verändert, kann Fig. 6 als Illustration des Gesagten dienen. In der Mitte schneidet, wie schou angedeutet, in dieses Schaltstick die mediane Trennungsfurche der Unterkieferbogenfortsätze noch etwas ein, hinten erhebt sich dasselbe etwa bis zur Höhe der Vereinigungsstelle der zweiten Kiemenbogen. Von dieser Vereinigungsstelle aus verläuft in der Mittellinie des Bodens der Mundhöhle eine ziemlich schmale und scharfe Leiste (mL. Fig. 6) nach hinten, die ein 
wenig dorsalwärts convex aufgebogen ist. In die hintere Kopfbeuge der Mundhöhlendecke springt das Ende dieser Leiste knopfförmig ein; bald unter der Spitze des Knopfes, an dem Abhange, der dem hintern Schenkel der Kopfbeuge gegenüberliegt, beginnt der schlitzförmige Eingang in den Kehlkopf, so dass die Falten, die denselben seitlich begrenzen, ztsammen die Fortsetzung des medialen Kammes bilden (vergl. Fig. 6 A l). An den Seitenrand des letzteren setzen sich von vorn nach hinten aufeinander folgend zuerst die zweiten Kiemenbogen, dann weniger stark nach hinten convergirend die kürzeren dritten, und zuletzt die noch kürzeren, vollkommen queren vierten an. Doch sind letztere nur schwer sichtbar zu machen, weil dieselben in den hintern Schenkel der hintern Kopfbeuge fallen; der Knickungswinkel des Bodens der Mundrachenhöhle liegt am Hinterrande der dritten Kiemenbogen. Der Ansatz der vierten Kiemenbogen findet also an dem hintern Abhange des oben beschriebenen medianen Knopfes statt, dicht vor und neben dem Beginn des Kehlkopfschlitzes. Zwischen den zweiten, dritten und vierten Kiemenbogenwülsten verlaufen in entsprechender Richtnng jederseits zwei tiefe Furchen bis an den medianen Kamm heran; es sind dies die zweiten und dritten inneren Kiemenfurchen. Dieletzteren fallen in den hintern Schenkel der hintern Kopfbeuge. Der hintere Rand derselben ist noch deutlich wulstig erhoben; dieser kurze Querwulst ist eben der vierte Kiemenbogen; eine hintere Begrenzung desselben konnte ich an dem sehr kleinen und engen Schlunde des Präparates selbst nicht mehr wahrnehmen, sehr wohl aber an dem Modell; ich komme darauf unten zurück. Ueher die in den innern Schlundfurchen gelegenen innern Kiemenöffnungen gibt nur das Modell sichern Aufschluss.

Ueber die Gefässverhältnisse ergiebt sich nach dem Modell und den Schnitten folgendes Bild, das mit dem von $\mathrm{H}$ is für seinen Embryo B in $B_{1}$ Taf. VII (Nr. I) construirten in den wesentlichsten Puncten übereinstimmt. Der Truncus arteriosus erreicht die ventrale Wand der Rachenhöhle am Vorderrande der zweiten Kiemenbogen und verläuft derselben angeheftet und etwas zur Oberfläche des Schlundbodens aufsteigend rückwärts bis vor den Grund der Keblkopfspalte, wo aus dem abgerundeten Ende des Gefässes zwei kleine divergirende Aeste hervorgehen, die den Anfang der Trachea umfassen und nach den Rickenaorten hinstreben, ohne dieselben zu erreichen; es sind dies die Anlagen der fünften Aortenbogen. 
Etwa in einer Querlinie mit dem hintern Rande der dritten Kiemenbogen steigt aus den Truncus arteriosus ein kurzer Ast, dessen Durchmesser dem Stamme kaum etwas nachgibt, senkrecht gegen die Schleimhantoberfläche auf; es ist dies der gemeinsame Abgang des 2., 3., 4. Aortenbogens. Derselbe theilt sich sogleich in zwei seitlich und etwas nach vorn divergirende starke Aeste, die im dritten Kiemenbogen gelegen um den Darm herum zur Rückenaorte jeder Seite streben. Ein kurzer Stamm biegt von derselben Theilungsstelle parallel der Schleimhautoberfläche nach vorn um, verläuft also gerade umgekehrt, wie der Truneus arteriosus selbst, und theilt sich, entprechend dem Ansatze des zweiten Kiemenbogens an die mediane Leiste, in zwei quer divergirende Aeste, die aber nicht mehr an den Seitenrand des Schlundes herumreichen; diese Reste der zweiten Aortenbogen entspringen also mit einem gemeinsamen ventralen Stamme. Aus derselben Theilungsstelle wie die dritten und der ventrale Stamm der zweiten entspringen endlich noch zwei starke Aeste, die um das innere Ende der dritten Kiemenspalte im Schlundboden schräg nach aussen und hinten laufen, in die vierten Kiemenbogen eintreten und ohne eigentliehe Krümmung die Rückenaorteu erreichen, welche selbst dem Schlunde parallel an der hintern Kopfbenge umbiegen.

Man sieht, wie vollkommen die Uebereinstimmung mit dem oben angezogenen Bilde von $\mathrm{His}$ ist.

Das Epithel der Mund-Rachenhöhle ist, wie schon die meisten Autoren hervorgehoben haben, ein niedriges, an vielen Stellen scheint es aus einer einfachen, im frischen Zustande wahrscheinlich mit Cilien versehenen Cylinderzellenlage zu bestehen; beim Uebergang in die Kiementaschen wird das Epithel mehrfach geschichtet. Knorpel ist in unserm Stadium noch nirgends vorhanden, das embryonale Bindegewebe erscheint sehr dichtkernig; um das Centralnervensystem liegen die Kerne lockerer, die Zellen zeigen deutliche Fortsätze und sind der Oberfläche desselben parallel stratificirt. Die Hauptnervenzïge sind in ihrer bekannten typischen Anordnung zu den Kiemenbogen und Kiemenspalten deutlich erkennbar. Ioh habe keine Veranlassung auf diese Dinge hier speciell einzugehen.

Aus den Schnitten und dem Modelle ergiebt sich über die speciellen Verhältnisse der Kiemenöffnungen, Kiemenspalteu u.s. w. Folgendes. Die Furche zwischen dem Schaltstücke $(\mathrm{Kx})$ and dem 
zweiten Kiemenbogen am Boden der Mundhöhle ist blind. Die erste Kiementasche öffnet sich nur in der Seitenwand der Mundhöhle und zwar mit einer langen Spalte über der keilförmigen Wurzel des Oberkieferfortsatzes, der iber dem seitlichen Ende des Unterkiefers nach innen vorspringt. Nach hinten reicht die Oeffnung bis an den zweiten Kiemenbogen. Von dieser Oeffuung aus verläuft die Kiementasche im Durchmesser von vorn nach hinten sich allmählich verengend und etwas winklig abgeknickt nach aussen und dorsalwärts.

Sie erreicht mit ihrem erweiterten Ende nur das oberste Ende der ersten äussern Kiemenfurche; nur an dieser Stelle verschmelzen die beiderseitigen Epithellagen.

Die Wurzel des Oberkieferfortsatzes, über welche sich die erste Kiementasche nach aussen erstreckt, nennt Moldenbauer (Nr. 7) beim Hibhnchen colliculus palatopharyngeus, die Furche, die denselben vom Unterkiefer trennt, sulcus lingualis, den Anfang der Kiementasche sulcus tubo-tympanicus.

Im Gegensatze zur ersten greifen die beiden nächsten inneren Schlundspalten-Oeffnungen weit auf den Boden der Mundrachenhöhle ïber. Die zweite stellt einen weiten, nach vorn leicht convexen Spalt dar, der dicht neben dem medialen Längskamme zwischen dem zweiten und dritten Kiemenbogen am Mundhöhlenboden beginnt und von der queren Richtung ein wenig nach hinten abweichend bis in die niedrige Seitenwand der Mundrachenhöhle einschneidet. Diese Oeffnung führt nun in die eigentliche Schlundtasche, einen eigenthümlich gestalteten Raum, von dessen Besonderbeiten bisher nur Wölfler eine annähernd richtige Vorstellung gehabt hat, die ihn aber dazu fiuhrte, von diesem Raum fälschlich die Thyreoidea abzuleiten. Fig. 4 zeigt diesen Hohlraum nach dem Modell ohne Epithelüberzug, also relativ zu weit. Derselbe erscheint als eine im queren Durchmesser abgeplattete Tasche, die mit einem verticalen Schenkel in der Hinterhälfte des zweiten Kiemenbogens gelegen ist, mit den Flächen (der medialen und der lateralen) der Aussenfläche dieses Bogens parallel gekrümmt, und mit einem andern Schenkel in die horizontale, dem Schlundboden parallele Richtung umbiegt und sich zwischen den Mundhöhlen-Erhebungen des zweiten und dritten Kiemenbogens nach innen erstreckt (zwischen $\mathrm{Kb}^{2}$ und $\mathrm{Kb}^{3}$ Fig 4).

Die oben erwähnte Oeffnung dieser Tasche in die Mund- 
Rachenhöhle nimmt weder die ganze Länge, noch die ganze Höhe ibrer Innenfläche ein, sondern beschränkt sich auf den vordern, grössern Theil des ventralen Schenkels und dem der Seitenwand des Schlundes entsprechenden Theil des verticalen; über den obern Rand des letzteren ist die Tasche noch blind ausgebaucht (der Richtung des weissen Pfeiles in Fig.4 folgend; vgl. auch o Fig. 10 Taf. XI). Die Berührung mit dem Epithel der zweiten äussern Kiemenfurche findet längs dem Hinterrande des verticalen Schenkels der Tasche in einer Linie statt, deren Länge ziemlich dem aiusserlich sichtbaren Theile jener Furche entspricht (zwischen $\alpha$ und $\beta$ in Fig. 4). Geht man also von der zweiten innern Kiemenöffnung aus ein, so gelangt man direct ventralwärts in einen blinden Raum, von der Oeffnung der Seitenwand aus nach oben ebenfalls in einen blinden Raum und nur von der Seitenwandöffnung aus uach hinten, die Länge der Kiementasche durchmessend, erreicht nan die sogenannte äussere Mündung. Nach dem Modell betrug, auf die Originalmaasse reducirt, die Breite der Mundrachenhöhle an der Stelle der zweiten innern Kiemenöffuungen $1,1 \mathrm{~mm}$, davon kamen am Boden auf die mittlere Leiste $0,5 \mathrm{~mm}$, auf die Spalten jederseits $0,3 \mathrm{~mm}$.

Die vordere Anlage der Thyreoidea fällt mit dem Vorderrande der zweiten innern Kiemenöffnnngen in eine Querlinie. Man findet dieselbe in der Medianebene gerade am Beginn der medianen Leiste, an die sich der Reihe nach die Kiemenbogen ansetzen. Die Anlage hat durchaus dasselbe Ansehen, wie die jeder andern Drise (vergl. Fig. $10 \mathrm{mS}$.). Aus einer kleinen Vertiefung zieht ein Epithelstrang ventralwärts (in der Länge von $0,1 \mathrm{~mm}$ ), der sich zu einer von hinten her löffelartig ausgehöhlten Epithelmasse verbreitert. - Die ausgehöhlte Mitte derselben ist sehr dünn, so dass es oft den Anschein hat, als theile sich der Epithelstrang in zwei bogenförmig divergirende Aeste. Im Innern der seitlichen Enden waren Lumina erkennbar.

Dicht an die concave Seite der Thyreoidea-Anlage reicht mit dem obern Rande das Ende des ventralen gemeinsamen Stammes der beiden rudimentären zweiten Kiemenarterien (II Fig. 5) heran. Dass diese Anlage wirklich die vordere Anlage der Thyreoidea ist, erhellt natürlich erst aus ibrer unten za schildernden, weiteren Entwicklung.

Die dritten innern Kiemenöffnungen schneiden wieder von der Seitenwand in den Boden der Mundhöhle bis zu dem medianen 
Kamm hin ein, der, wie Fig. 5 zeigt, hier steiler abfällt, als im Bereiche der zweiten Kiemenspalten. Der Hohlraum der dritten Kiementasche hat eine ähnliche Form, wie der der zweiten, indem auch er einen verticalen und einen horizontalen Schenkel zeigt. Seine Längsausdehnung (im Durchmesser von vorn nach hinten) ist bedeutend geringer, so dass die innere Kiemenöffnung beinahe die ganze mediale Wand einnimmt. Nur eine kleine, blinde Ausstiilpung ragt ïber die Oeffnung an der Seitenwand nach oben in die Substanz des dritten Kiemenbogens hinein; diese Ausstülpung ist noch durch eine bei $\gamma$ Fig. 5 sichtbare Leiste besonders abgesetzt. Der horizontale Schenkel der Kiementasche ist ventralwärts blind geschlossen; die Oeffnung in die äussere Kiemenfurche findet an der Uebergangsstelle der beiden Schenkel (zwischen $\alpha$ und $\beta$ Fig. 5) statt. Von dem medialen Ende des horizontalen Schenkels zieht eine jetzt noch kurze blinde Tasche ventralwärts und zugleich nach innen und etwas nach vorn (in der Richtung des weissen Pfeils in Fig. 5). Sie geht hinter der Abgangsstelle des dritten und vor der des vierten Aortenbogens aus dem gemeinsamen Stamme des zweiten, dritten und vierten Aortenpaares quer vorbei; ihr blindes Ende reicht bis in die Nähe der Pericardialhöhle. Diese Ausstülpung ist mit geschichtetem Epithel überzogen und zeigt ein deutliches Lumen; es ist dies, wie sich weiterhin ergibt, die epitheliale Anlage der Thymus. - Von einer vierten Kiementasche war bei unserm Schweinsembryo nur ein inneres Rudiment vorbanden, das sich durch Vergleich mit einem ältern Stadium, wo zufällig die Verbindung dieses Rudiments mit einem von der vierten äussern Kiemenfurche eingesenkten Epithelstrange nur eine ganz kleine Strecke weit unterbrochen war, mit grosser Wahrscheinlichkeit als solches diagnosticiren liess. An dem hintern Schenkel des der Nackenbeuge entsprechend geknickten Rachenhöhlenbodens findet man zu Seiten der Längsfalten, die die schlitzförmige Kehlkopfspalte begrenzen, zwei ventralwärts und etwas nach aussen und hinten gerichtete Einsenkungen, die sich wie Drüsenanlagen ausnehmen; ihr blindes Ende liegt jederseits hinter den vierten Aortenbogen. Ein Bild derselben freilich aus weiter vorgeschrittenen Stadien gibt Fig. 12 Taf. XI 1S. - Durch diese Einsenkungen werden die vierten Kiemenbogen an der Schlundoberfläche einigermassen nach hinten begrenzt. Ueber ibre Schicksale und Bedeutung unten ein Weiteres. 


\section{Embryonen von $11 \mathrm{~mm} \mathrm{~N}$. L.}

Der Entwicklung der Extremitäten nach etwas älter, wie die menschlichen Embryoneu von $\mathrm{H}$ is $\mathrm{A}$ und $\mathrm{B}$ (Nr. 1); die Basis der obern Extremität ist nur wenig eingeschnürt; ihre Spitze ist etwas aboralwärt:s gewendet, so dass ihre Axe nunmehr mit der Rückenkante einen spitzen Winkel bildet. Während im vorigen Stadium die Rückenfläche der obern Extremität fast ohne Absatz in die Oberfläche des Stammtheils überging, hebt sich jetzt die Basis der Extremität wulstartig heraus; es entsteht ein kurzes keilförmiges Stück, das mit seiner Längsaxe senkrecht auf der Fläche des Körpers steht, und in einem rechten Winkel in das Hauptstück umbiegt, dessen Flächen der Körperwand annähernd parallel liegen. Es ist dies die erste Andeutung der Gliederung der Extremität, der rechte Winkel entspricht wahrscheinlich dem Ellenbogen; ein ähnliches Stadium liegt offenbar His Fig. 2 l. c. Taf. I zu Grunde. Die hintere Extremität ist wieder weiter zurück, wie die vordere. Die Nase ist taschenförmig; die Thränenfurche tief u. s. f.

Es sind noch alle Kiemenbogen sichtbar, wie im vorigen Stadium; doch nicht bei allen Exemplaren der Tracht in gleicher Weise. Bei dem abgebildeten Embryo (Fig. 2) ist der dritte stark unter das Flächenniveau der Umgebung eingesunken, beinahe ebenso tief, wie der vierte; er liegt mit diesem zusammen in einer Vertiefung, die von scharf abfallenden Rändern umgeben ist. Namentlich der dorsale Umschlagsrand, der in der Verlängerung des im vorigen Stadium den vierten Kiemenbogen begrenzenden Randes gelegen ist (vergl. Fig. 1 mit Fig. 2), ist scharf. Er grenzt die Gegend des dritten und vierten Kiemenbogens von der jetzt deutlich ausgeprägten Wolff'schen Leiste ab, die von den Wurzeln der ersten beiden Bogen gegen die Extremitäten-Anlage herabzieht. Zugleich bemerkt man, dass der dritte Kiemenbogen in allen Dimensionen, namentlich aber im oral-aboralen Durchmesser (wenn man den Bogen als Längsspange für sich betrachtet "in der Höhe") sowohl relativ, wie absolut kleiner geworden ist. Ebenso hat der vierte Kiemenbogen abgenommen. Hier folgen die ungefähren Maasse in Millimetern:

I. Kiemenbogen $\left\{\begin{array}{ccc}\text { Höhe des dorsalen Endes } & 0,53 \\ , \text {, ventralen } " & 0,8\end{array}\right.$

Länge der I. äussern Kiemenfurche 1,1

II. Kiemenbogen $\left\{\begin{array}{ccc}\text { Höhe des dorsalen Endes } & 1,2 \\ , \quad \text {, ventralen }, & 0,5\end{array}\right.$

Länge der II. äussern Kiemenfurche $\quad 0,66$ 


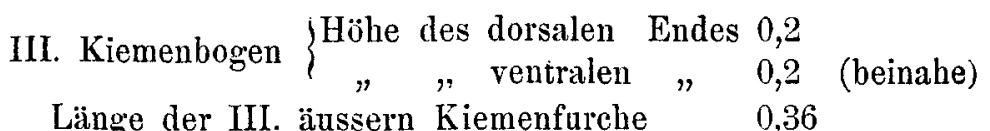

Visst man in Fig. 1 und Fig. 2 die Entfernung zwischen der ersten und dritten äussern Kiemenfurche, so findet man, dass dieselbe fast genau die gleiche geblieben ist; die Höhenzunahme des zweiten Kiemenbogens hat zu einer Ueberlagerung des dritten geführt, der seinerseits schon früher den vierten zu überlagern begonnen hat. Der untere Rand des zweiten Kiemenbogens ist nicht mehr grade, sondern springt convex nach hinten vor, dabei hat sich eben der hintere Theil desselben klappenartig über die Aussenfläche des dritten Bogens vorgeschoben und bildet so den Kiemendeckelfortsatz der Autoren. Der erste und ihm folgend der zweite Kiemenbogen sind auch im dorso-ventralen Durchmesser stark gewachsen und haben sich unter dem Schädel nach vorn vorgeschoben. Ihre Aussenfläche erscheint nun bedeutend stärker gewölbt. Die ventrale Randcontur der Kiemenbogen liegt in Fig. 1 vom vierten bis zum vordern Ende des zweiten beinahe in einer Geraden und weicht vom ersten an nur wenig ventralwärts ab. Ihre Verlängerung schneidet ziemlich dicht vor dem Augenbügel durch. Dagegen springt der ventrale Rand der beiden ersten Kiemenbogen ïber den der beiden letzten in Fig. 2 merklich vor; die Verlängerung der ventralen Randcontur derselben würde weit vor dem Auge vorbeigehen. - Bei andern Exemplaren dieser Tracht waren die Veränderungen am dritten Kiemenbogen viel weniger weit vorgeschritten, er war kaum merklich eingesenkt und hatte sogar an Höhe vielleicht noch eine Spur zugenommen. Das Wulstrelief am ersten Kiemenbogen ist im vorliegenden Stadium viel ausgeprägter als im vorigen. Eine vierte äussere Kiemenfurche war kaum noch zu erkennen, obgleich die Schnittuntersuchung an der hintern Spitze des kleinen Dreiecks, das in Fig. 2 den vierten Kiemenbogen repräsentirt, eine tiefere Einziehung and einen vom Grunde derselben medialwärts in's Bindegewebe eindringenden Epithelstrang kennen lehrte, der der Ausstiilpung lS Fig. 12 der seitlichen Schlundwand sehr nahe kommt und mit derselben wohl einen Rest der vierten Schlundspalte darstellt (siehe unten). Doch war dieses Verhältniss nur an dem einen Exemplar, dem Fig. 2 entnommen ist, einigermassen deutlich. Fig. 6 lehrt, dass das Relief des Nundhöhlenbodens in seinen Hauptzügen noch ebenso 
gestaltet,ist, wie im vorbeschriebenen Stadium; einzelne Abänderungen der Dimensionsen und einzelne Lageverschiebungen sind nachzutragen. Die oben erwähnte Vorschiebung des Unterkieferfortsatzes unter dem Schädel hinweg nach vorn kommt wesentlich auf Rechnung des Schaltstïckes, das sich sowohl vom Unterkieferbogen wie vom zweiten Kiemenbogen am Mundböhlenboden schärfer absetzt, als bisher. Es betrug nach einer Messung am Präparate selbst, so genau wie eine solche ausführbar war:

im vorigen Stadium die Länge des Schaltstückes $0,4 \mathrm{~mm}$,

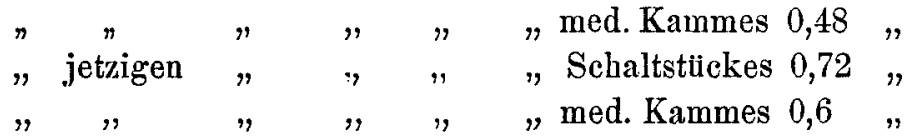

Der mediane Kamm, der zwischen den Ansätzen der Kiemenbogen gelegen ist, hat sich nicht bloss, wie die angeführten Zahlen zeigen, verlängert, sondern auch in seiner Form verändert; binten erscheint derselbe merklich verbreitert und erhöht, vorn seitlich geht er allmählicher in die stark erhobenen zweiten Kiemenbogen über. Die Furchen zwischen dem zweiten bis vierten Kiemenbogen zeigen sich noch deutlich, erscheinen aber doch seichter wie friiher.

Die dritten Aortenbogen sind viel schwächer geworden, namentlich auf der rechten Seite; ganz dünn sind die dorsalen Verbindungen des dritten und vierten, am stärksten erscheint der vierte linke Aortenbogen. Die wichtigste Veränderung jedoch betrifft die Abgangsstelle des gemeinsamen ventralen Stammes der fünften Bögen. Während dieser im vorher besprochenen Stadium die direkte Verlängerung des Aortentruncus nach Abgabe des kurzen aufsteigenden gemeinsamen Stammes des zweiten bis vierten Bogens bildete, so dass der Anfang des gemeinsamen Stammes der 5. Bogen im ventralen Schlundboden lag, hat sich jetzt die Scheidung zwischen dem gemeinsamen Stamm der zweiten bis vierten Aortenbogen und dem der fünften bis in das Pericard hinein fortgesetzt, d. h. der truncus arteriosus spaltet sich jetzt nach kurzem einfachen Verlauf in zwei starke Zweige, der vordere tritt sogleich im Bereich des dritten Kiemenbogens dorsalwärts in den Mundhöhlenboden, sein stärkster Ast, der also als direkte Fortsetzung zu betrachten ist, ist der linke vierte Aortenbogen; mit ihm zusammen stellt er später nach vollkommener Trennung $\mathfrak{u}$. s. f. den aufsteigenden und den Bogentheil der bleibenden Aorte dar; der andere Zweig verlässt das Pericard weiter rückwärts und umgreift mit zwei, jetzt stark gewor- 
denen Aesten Trachea und Oesophagus, um die absteigenden Rückenaorten zu erreichen; dieser Stamm ist die Anlage der Art. pulmonalis.

Die inneren Kiemenöffinungen sind keineswegs mehr in der vorigen Ausdehnung erhalten. Die zweite Kiementasche öfnet sich fast nur mehr in der Seitenwand der Mundhöhle; - beinahe der ganze horizontale Schenkel derselben (siehe oben) und mit ihm die in denselben führende Spalte am Mundhöhlenboden zeigen sich verlegt; nur am lateralen Rande des Mundhöhlenbodens vertieft sich die zweite innere Kiemenfurche noch, wie früher, zu einer kleinen horizontalen Fortsetzung der eigentlichen Kiementasche. Der jetzt noch bestehende Rest der Kiementasche ist ein wesentlich sagittaler (kaum in den Mundhöblenboden umgebogener), der Oberfläche des Kiemenbogens parallel abgeplatteter, enger Spalt, der nach wie vor mit einer blinden Ausstillpung dorsalwärts über die Decke der Mundhöhle hinausragt. Die Einmïndung in die Mundhöhle liegt an seinem vorderen Ende, wo derselbe in dorso-ventraler Richtung am höchsten ist; nach hinten zieht er sich niedriger werdend lang aus $(11 / 40$ gegen $6 / 40 \mathrm{~mm}$ bei dem vorigen, nach Schnittdicken gemessen) und erreicht die zweite äussere Kiemenfurche nur noch an einer sehr circumscripten Stelle. Zweiter und dritter Kiemenbogen sind also jetzt neben dem medianen Kamme am Mundhöhlenboden solid verbunden.

Die epitheliale Anlage der Thyreoidea hat sich von ihrem Mutterboden abgelöst, der Stiel, der dieselbe mit dem Mundhöhlenepithel verband, ist verschwunden; im übrigen behauptet sie noch dieselbe Stellung am vordern Ende des ventralen zweiten Kiemenarterienstammes, wie früher; auch zeigt dieselbe noch eine ähnliche Form, nur ist der mittlere Theil stärker geworden und erscheinen die seitlichen Schenkel mebr quer gestellt. Die von His als Art. linguales angesprochenen Fortsetzungen der ventralen Stücke der zweiten Aortenbogen, welche die Anlage umgreifen, fand ich jetzt deutlicher.

Die dritte innere Kiemenöffnung und die dritte Kiemenspalte haben sich in ziemlich complicirter Weise verändert. Die in den Boden der Mundhöhle medianwärts einschneidende Tasche (Fig.5) hat sich an der Einmündung verlegt, der in der Tiefe offen bleibende Raum dient der Thymusanlage als Einmündungsrohr in den Rest der Kiemenspalte, welcher letzterer nun von der Oeffnung in 
der Seitenwand der Schlundhöhle aus schräg lateral- und ventralwärts und zugleich etwas rïckwärts bis zum ventralsten Ende der dritten äussern Kiemenfurche zieht. Im Bereich der blinden dorsalen Aussackung der dritten Kiementasche hat sich die vordere Wand an die hintere gelegt. So entsteht dort ein grosses Epithellager, das ventralwärts mit dem noch gangbaren Theil der Kiementasche, nach innen mit der seitlichen Schlundwand und nach aussen mit dem Epithel des dorsalen Theiles der dritten äussern Kiemenfurche zusammen hängt.

Die Thymusanlagen haben sich erheblich verlängert, zeig*en aber in Bezug auf Lage und Aussehen dasselbe wie friher. Ihre blinden Enden liegen zur Seite des Austritts des ventralen Aortenstammes aus dem Pericard; sie behalten diese characteristische Beziehung. noch sehr lange.

Gerade an der Schnittserie, die durch den der Figur 2 zu Grunde liegenden Kopf gelegt wurde, zeigte die ventrale Ausstïlpung der seitlichen Rachenwand, die zur Seite des Kehlkopf'schlitzes gelegen ist und nach dem oben Ausgefiihrten die hintere Grenze des vierten Kiemenbogens bezeichnet, eine Beziehung, die dieselbe als inneren Rest der vierten Kiementasche zu deuten erlanbt. Ganz nahe an ihren blinden Grund reichte nämlich ein Epithelstrang, der von dem dorsalen Ende der nur angedeuteten vierten äussern Kiemenfurche in die Tiefe zieht; obgleich beide Gebilde im Winkel zu einander standen, ist ein früherer Zusammenhang äusserst wahrscheinlich. Der blinde Grund der Einsenkung selbst erschien hie und da, wie bei der Bildung von acinis, mit Buckeln besetzt.

\section{Embryonen von 13 mm Nackenlänge.}

Diese Schweinsembryonen entsprechen den menschlichen von $8-10 \mathrm{~mm}$ bei $\mathrm{H}$ is (Nr. 2) in vielen Punkten. An den Extremitäten hat sich "eine scheibenförmige Endplatte als Anlage von Hand und Fuss vom Wurzelstücke abgegliedert." An der untern ist die Veränderung jedoch erst im allerersten Beginn. Die Nackenkrümmung beträgt einen rechten Winkel.

An der Kiemenbogengegend fallen folgende Veränderungen ins Auge: Der ventrale. Rand des ersten Kiemenbogens hat sich mit demselben Rande des zweiten durch eine Bricke in Verbindung gesetzt. Es geschieht dies beim Schwein nicht in der Weise, wie es $\mathrm{His}$ vom menschlichen Embryo schildert (Nr. 2 p. 56), wo es 
heisst, dass das untere Ende des Kinnwulstes des Unterkieferfortsatzes sich lappenförmig nach aufwärts und rückwärts ausbreitet und die Spalte, sowie den untersten Höcker des zweiten Schlundbogens zudeckt. Der dritte (ventralste) Höcker des zweiten Schlund. bogens ist im Gegentheil in Fig. 3 noch deutlicher sichtbar, als in Fig. 2 und zwar dadureh, dass sich der denselben umgebende, ventrale Theil der ersten äussern Kiemenfurehe in eine breite Grube verwandelt bat. Die A usbildung einer Substanzbrïcke um den untern Rand der ersten äussern Kiemenfurche herum scheint vielmehr lediglich mit dem ansgiebigen dorso-ventralen Wachsthum des ersten und zweiten Kiemenbogens zusammenzuhängen, mit dem die Ausdehnung der Kiemenfurche in derselben Richtung nicht gleichen Schritt hält; darauf weist anch die jetzt convex vorspringende gemeinsame ventrale Profilenntour der beiden Bogen hin (vergl. Fig. 3). Die Wurzelhöhe des zweiten Kiemenbogens beträgt jetzt $1,8 \mathrm{~mm}$. Eine vollkommene Ueberlagerung der Aussenfläche des dritten und vierten Kiemenbogens findet auch jetzt nicht statt. Die Modelle und Schnitte weisen mit Sicherheit nach, dass das kleine dreieckige Feld, das hinter dem dorsalen Anfange des ventralen Randes des zweiten Kiemenbogens in Fig. 3 zu sehen ist, den Rest der Aussenfläche der beiden letzten Bogen repräsentirt, nur dass die Furchen, die sie von einander und den hinten angrenzenden Bezirken trennten, fast ganz geschwunden sind. Von der scharfen Kante, die in Fig.2 den dorsalen Rand der Aussenfläche der beiden letzten Bogen begrenzt, ist in Fig. 3 noch ein deutlicher Rest zu sehen. Bei etwas grösseren Embryonen schwindet diese Kante. Bald darauf hat die Rückenkrümmung. (zum zweiten Male?) ihr Maximum erreicht. $\mathrm{Zu}$ dieser Zeit ist der hintere (frither rentrale) Rand des zweiten Kiemenbogens am schärfsten und legt sich in Form einer dentlichen Falte nach hinten ïber jenes kleine dreieckige Feld hinweg, so dass er direkt an den Anfang der Herzerhebung angrenzt. Mit der Abnahme der Nackenkrümmung und der Anfrichtung des Koptes sehwindet diese Knickungsfalte vom dorsalen Ende her, der Rest der Aussenfläche des dritten und vierten Kiemenbogens wird frei und geht ohne Abgrenzung in den dorsalen Theil des zweiten Kiemenbogens über, der hinter der Ohröffnung gelegen ist. Die Entwicklung der ventralen Halsfläche und das Zurückweichen des vordern Herzendes babe ich nicht weiter verfolgt.

Zum Verständniss des Folgenden muss ich hier vorausgreifend 
die Veränderungen besprechen, die im Verlaufe der Entwicklung die Biegungen der Axe der Mund-Rachenhöhle erleiden.

Der Sattelwinkel der Mundhöhlendecke beträgt an Medianschnitten gemessen

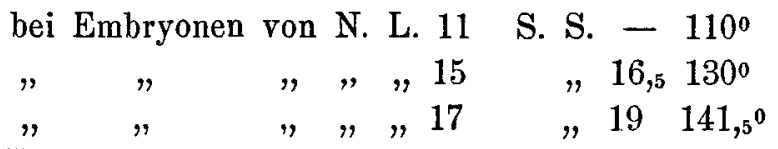

Bei Embryonen von mehr als $22 \mathrm{~mm}$ ist der Winkel in eine flache Rundung iibergegangen. Mit der rasch fortschreitenden Ausbildung des Ethmoidaltheiles des Kopfes tritt vor dem Sattelwinkel eine neue Knickung der Decke der Mundhöhle auf, die Sphenoethmoidalbeuge Dursys. Der Ethmoidaltheil wird dabei ventralwärts abgebogen. Bei Embryonen von $15 \mathrm{~mm} \mathrm{~N}$. L. beträgt dieselbe

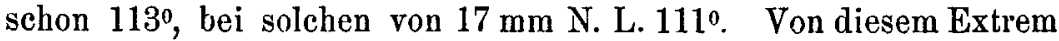
nimmt der Winkel allmählich wieder zu. Wie verbält sich zu diesen Veränderungen der Boden der Mundrachenhöhle? Derselbe schiebt sich, wie oben schon erwähnt, vor und erhebt sich gleichzeitig wulstartig. Während bei Embryonen von $7 \mathrm{~mm}$ der Vorderrand des Unterkieferbogens nur den Bezirk der Decke dicht vor dem Sattelwinkel erreicht, rückt derselbe allmählich bis zum Vorderrande des Ethmoidaltheils, der dabei ganz unverhältnissmässig in die Länge wächst, vor. Der ganze Raum, der von dem Sattel- und Sphenoethmoidalwinkel umschlossen ist, wird durch eine Erhebung am Boden der Mundhöhle ausgefüllt, die gleich hinter dem Rande des Unterkieferfortsatzes beginnt und sich bis zur hintern Kopfbeuge erstreckt; es ist dies die Zunge. An der Bildung derselben participiren, wie ich vorausnehmen muss, in erster Linie das Schaltstiick, das den 'Zungenkörper' liefert, in zweiter die zweiten Riemenbogen mit dem zwischen ihren medialen Enden gelegenen Theile des medianen Längskammes. Das Genauere darüber folgt sogleich.

Die specielle Verfolgung der Entwicklung des Daches der Mundhöhle liegt ausser dem Bereiche meiner Aufgabe.

Kehren wir jetzt zur Betrachtung des Mund-Rachenhöhlenbodens an einem Embryo von $13 \mathrm{~mm} \mathrm{N.} \mathrm{L.} \mathrm{zurück,} \mathrm{so} \mathrm{sieht} \mathrm{man,}$ dass das Schaltstiick, das bisher im Ganzen unter dem Nivean des zweiten Kiemenbogens lag, sich mit demselben zu einem einbeitlichen Wulste erhoben hat; die Spitze dieses Wulstes hat sich über die ventralwärts abgebogenen Unterkieferfortsätze hinweggelegt (vergl. Fig. 7, $\mathrm{Kx}$ und $\mathrm{Kb}$ ), während in Fig. 6 das Spaltstiick 
tiefer lag als die leistenförmig vorspringenden Unterkieferfortsätze. Die Länge des Schaltstückes ist von 0,72 auf $1,2 \mathrm{~mm}$ gestiegen. Die Vergrösserung desselben schreitet in späteren Stadien noch viel rascher vorwärts, denn dieser Theil liefert allein den Körper der Zunge (vergl. Fig. 6-9 Kx). Gegen die zweiten Kiemenbogen grenzt sich das Schaltstiuck mit zwei queren Wulsten ab, die in erster Andeutung schon im vorigen Stadium zu sehen waren (vergl. Fig. 6 u. Fig. 7); weiter vorn sieht man zwei längsgestellte Erhabenheiten, die einen schmalen medianen Kamm zwischen sich fassen. Diese letzteren fallen nach vorn zu den Unterkieferfortsätzen steil ab, der steile Abhang liegt dem Ethmoidaltbeil der Decke an. Der mediane Kamm drängt sich bei ältern Embryonen zwischen den seitlichen Erhabenheiten heraus und wächst mit ihnen zu einem einheitlichen Körper nach vorn und ventralwärts frei beraus und bildet so den freien Theil der Zunge (vergl. Fig. 8). Das Wachsthum dieses letzteren eilt, wie Fig. 7 bis 9 lehren, dem des festsitzenden Theiles in nächster Zeit ungemein voraus. - Die zweiten Kiemenbogen sind in Fig. 7 noch deutlich abzugrenzen, ihre Richtung ist eine mehr quere geworden, zwischen ihnen ist der verschmälerte mediale Kamm noch schwach erkennbar. Die Furchen am vordern Rande sind undeutlicher als am hinteren; in den folgenden Stadien wird, wie noch zu besprechen, die Verschmelzung mit dem Schaltstiicke eine innigere; immer deutlicher bilden beide zusammen die einheitliche Erhebung der Zunge; -- die zweiten Kiemenbogen mit ihrem medialen Verbindungsstuicke liefern die Zungenwurel. Die dritten Kiemenbogen $\mathrm{Kb}_{3}$ erseheinen in Fig. 7 sehr verschmälert, dagegen hebt sich ihr medianes Verbindungsstück zusammen mit dem der vierten Bogen stark hervor und grenzt sich bald darauf nach vorn gegen das der zweiten durch eine winklige Furche scharf ab (vgl. Fig. 7 mit Fig. 8). Sehr bald erscheinen die dritten Bogen, die von dem lateralen Ende her verschwinden, wie zwei kleine Querflügel des Mittelstückes. Der Theil der medianen Leiste zwischen dritten und vierten Kientenbogen wird, wie ich vorausschicken muss, zum Kehldeckel (Eg Fig. 7-9). Als hintere Grenze der Reste der dritten Kiemenbogen sind noch zwei Eindriicke erkennbar. Die vierten Kiemenbogen konnte ich nicht mehr als gesonderte Erhebungen erkennen, sie gehen in der schmalen ventralen Rachenwand auf, die zur Seite des Kehlkopfschlitzes und der Arytänoidfalten liegen. 
Aus den Schnitten ergibt sich noch deutlicher, wie aus den Flächenansichten, dass es nicht das ganze Schaltstiick und die ganzen ventralen Theile der zweitęn Kiemenbogen sind, die sich zur Zunge erheben, sondern dass um dieselbe ein rinnenartiger Raum vertieft bleibt, der sich nach hinten gegen die Zungenwurzel (zweiten Kiemenbogen) hin in bekannter Weise verflacht.

Von den Gefässveränderungen will ich nur erwähnen, dass die dorsalen Verbindungen der dritten und vierten Aortenbogen jetzt ganz verschwinden; auf weitere für mich wichtige Einzelheiten komme ich unten bei der Thyreoidea zurück.

Die erste Kiementasche, sei nebenbei erwähnt, erreicht jetzt die epitheliale Auskleidung der ersten äussern Schlundfurehe nicht mehr. Im zweiten Schlundbogen erseheinen zwei strangartige Verdichtungen, die die lateralen Theile der Mundrachenhöhle von der ventralen Seite her umfassen; es sind die Anlagen der kleinen Znngenbeinhörner, die noch etwas eher, wie die der Merkel'schen Knorpel, aufzutreten scheinen.

In der niedrigen Seitenwand der Rachenhöhle findet man an Hinterrande des zweiten Kiemenbogens die sehr verkleinerte innere Oeffinung der zweiten Schlundspalte. Der Anfang der letzteren zeigt einen unbedentenden Rest der blinden, dorsalwärts gerichteten Ausstuilpung; im Uebrigen ist der früher ausgedehnte Raum in einen engen Kanal und weiter nach rückwärts in einen soliden Epithelstrang verwandelt, der schliesslich aber noch die Epidermis erreicht - etwa an der Stelle, zu der der Strich von 2 in Fig. 3 führt, also nahe dem dorsalen Ende des scharfen Hinterrandes des zweiten Kiemenbogens. Dicht hinter der Einmiindung der zweiten Schlundspalte in die Rachenhöhle biegt, wie man jetzt sebr schön ïbersehen kann, die Anlage des Nervus glossopharyngeus von der dorsalen auf die ventrale Schlundseite.

Die quergestellte, halbmondförmige, vordere Anlage der Thyreoidea hat sich noch mehr in die Tiefe gesenkt. Während dieselbe im ersten Stadium weit vor dem Beginn des Kehlkopfschlitzes gelegen war, rückt sie diesem jetzt immer näher, später liegt sie ihm ventralwärts an und riickt schliesslich sogar hinter die Keblkopfanlage (vergl. mS Holzschnitt $b-d$ ).

Es sei mir erlaubt, hier diejenigen Monente im Zusammenhange aufzuzählen, die mir bei dieser, zuerst von W. Müller gewürdigten Verschiebung der auch die Ursprungsstellen der Aortenbogen und die Enden der Thymus- 
schläuche folgen, wirksam zu sein scheinen. In erster Linie ist der Umstand zu nennen, dass die Parthien des Bodens der Mundrachenhöhle, welche in Ebenen dorsalwärts von der Thyreoidea-Anlage liegen, viel stärker wachsen, als die ventralen Parthien. Man kann sich die Folgen dieser Erscheinung, sowie den Einfluss, den das vorwiegende Wachsthum der vordern Theile des Mundhöhlenbodens auf die Verschiebung der epithelialen Thyreoidea-Anlage hat, in folgender Weise klar machen: Stellt man an dem Bilde eines Medianschnittes, den man durch den Mundhöhlenboden eines Embryo von $11 \mathrm{~mm} \mathrm{~N}$. L. gelegt hat, die vordere Hälfte der geknickten Schnittlinie horizontal, so fällt die hintere

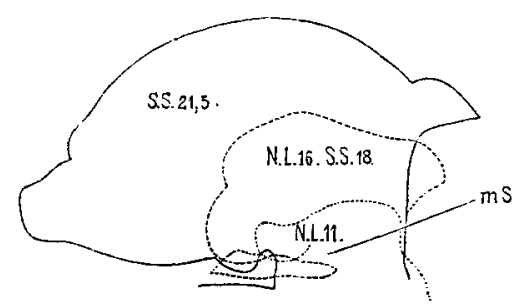

Drei amfeinandergclegte Contourzeichnungen von Median. schnitten durch den Mundhöhlenboden verschieder grosser Embryonen. Die Grösse derselben siehe in den Figuren. Vergr. 9 fach.

Hälfte mit dem Kehlkopfeingang in die Vertikale. Bezeichnet man auf diesem Bilde (Holzschnitta) durch einen Punkt die ungefähre Lage der vorderen Thyreoidea-Anlage, so sieht man, dass dieselbe sich weit vor dem Kehlkopfeingange, ungefähr in derselben Horizontalebene wie dieser, findet. Nun lege man analoge Durchschnittszeichnungen von Embryonen von $16 \mathrm{~mm}$ N. L. und $21,5 \mathrm{~S}$. S. in der Weise auf die erste Figur, dass die Durchschnittslinien der äussern Haut des Mundhöhlenbodens in dieselbe Horizontale und die des Kehlkopfeingangs in dieselbe Senkrechte fallen (vergl. Holzschnitt a), so sieht man, wie bei den der Aufeinanderlagerung der Figuren zu Grunde liegenden Wachsthumsbedingungen, die vordere Thyreoidea-Anlage sich am Kehlkopf vorbeischiebt und endlich vor den Anfang der Trachea zu liegen kommt. Die Voraussetzung der Aufeinanderlagerung der drei Durchschnittszeichnungen ist aber zunächst ein vollständiger Stillstand im Wachsthum seitens der ventral und hinter der Thyreoidea-Anlage gelegenen Parthien; ein solcher findet aber selbstverständlich nicht statt; diese Figuren sollen den Vorgang auch nur qualitativ illustriren, nicht quantitativ erschöpfend darstellend. Weitere Eactoren, die dabei einwirken, sind das vorzüglich nach hinten gerichtete Eigenwachsthum der Drüsenanlage, weiter eine Atrophie der hinter derselben gelegenen Parthien: - der unpaare ventrale Stamm der zweiten Aortenbogen schwindet und die Drüsenanlage kommt bei $13 \mathrm{~mm}$ langen Embryonen dicht vor die Abgangsstelle der dritten Aortenbogen aus der directen Fortsetzung des Truncus arteriosus in den linken vierten Aortenbogen zu liegen (vergl. Holzschnitt b) - endlich kommt auch die Neubildung der ventralen Halswand, sowie die Rückwärtswanderung des ganzen vorderen Herzens dabei in Betracht.

Die vordere Anlage der Thyreoidea findet sich bei Embryonen 
von $13 \mathrm{~mm} \mathrm{~N}$. L. in einer Querlinie mit den vordern Enden der Thymus-Schläuche. Lateral neben der vordern Thyreoidea-Anlage und ebenso lateral von den Thymus-Schläuchen zieht in sagittaler Richtung jederseits ein starker Nerv, dessen etwas der Mittellinie zugeneigtes vorderes Ende im Mundhöhlenboden noch bis in den Bereich des ersten Kiemenbogens erkennbar bleibt. Ruickwärts biegt der Nerv in Bogen dicht um die hintere Seite der dritten Kiemenspalte nach der dorsalen Seite des Schlundes herum und vereinigt sich neben dem Kehlkopfeingang mit der Hauptnervenmasse der VagusGruppe. Es ist kaum möglich denselben anders, wie als Hypoglossus, zu deuten.

Die vordere epitheliale Anlage der Thyreoidea hat sich namentlich in die Quere ausgedehnt, dieselbe ist mit zahlreichen Hervorragungen besetzt, im Innern sind aber noch keine Gefässe zu erkennen.

Die dritte Kiemenspalte durchbricht mit einer nur sehr kleinen Oeffnung die niedrige laterale Schlundwand am hintern Rande des lateralen Endes des verschmälerten dritten Kiemenbogens. Die Stelle fällt in denselben Schnitt, wie der Anfang der Kehlkopfspalte, es scheint sich demnach auch die Mitte des Rachenbodens im Vergleich zu den Seitentheilen mebr gehoben za haben. Die früher geräumige dritte Kiemenspalte ist jetzt in einen engen Kanal verwandelt, der von der queren Richtung etwas nach hinten abweicht. Lateralwärts geht derselbe in einen breiteren, soliden Zellstrang über, der nahe am obern Ende des scharfen Hinterrandes des vertieften dreieckigen Feldes (Fig. 3) dicht hinter der Ausmündung der zweiten Kiemenspalte mit der Epidermis zusammenhängt. Die innern Oeffnungen der zweiten und dritten Kiemenspalten sind immer noch reichlich $1 / 3$, die äussern kanm 1/8 mm von einander entfernt. Dicht an der Rachenmündung umgiebt den offenen Rest der dritten Schlundspalte ein namentlich dorsalwärts ansgedehntes Epithellager (bis 0,24 mm Höhe an manchen Schnitten), das offenbar mit dem schon im vorigen Stadium erwähnten und als Rest der dorsalen blinden Ausbuchtung der dritten Kiementasche gedeuteten Epithellager identisch ist, nur ist dasselbe mit der Zunahme des Breitendurchmessers weiter von der Hautoberfläche abgerijekt. In das ventrale, dreieckig zugespitzte Ende dieses Epithellagers mündet die schlauchförmige Thymus-Anlage ein; die friilher offene Einmindung derselben (vergl. oben p. 278) in den Kiemenkanal scheint solid geworden zu sein und die er- 
wähnte dreieckige ventrale Spitze des Epithellagers zu bilden. Die Thymusschläuche sind länger geworden, haben sich aber sonst in keinem wesentlichen Puncte verändert. Aus dem dritten Kiemenkanale zieht nahe der Schlındmïndung eine anfangs durchgängige, aber rasch blind endende Ausstülpung mach hinten, mitten in die Hauptnervenmasse der Vagus-Gruppe hinein. Dieselbe schwindet später ohne Spuren zu hinterlassen.

Die ventralen Ausstïlpungen der seitlichen Schlundwand, die oben als Reste der vierten Kiemenspalten gedeutet wurden, haben sich verlängert und sind jetzt medialwärts concav gekrümmt. Sie zeigen ein deutliches Lumen und mitunter am blinden Ende einzelne Höcker - wie zur Bildung von acinis. Dieselben behalten ihre charakteristische Lage hinter den vierten Aortenbogen (der bleibenden Aorte linkerseits, der Art. subclavia rechterseits); sie stellen, wie unten näher auszuführen, die paarigen hintern Thyreoidea-Anlagen dar.

\section{Aeltere Embryonen.}

Bei Schweinsembryonen von 14 NL. ist das beschriebene Relief der Zungenoberflïche noch gerade zu erkennen; - der vordere Theil, der vom Schaltstiick stammende Zungenkörper, ist bedeutend gewachsen und das vordere Ende desselben ein wenig frei. Eine wichtigere Veränderung hat sich am vordern Ende der Keblkopfspalte vollzogen. Während dieselbe bisher einen schmalen Längseinschnitt darstellte, erweitert sich der grössere vordere Theil derselben jetzt za einer queren, nach vorn convex gekrümmten Spalte, die das knopfförmige Ende der medianen Leiste, die Kehldeckelanlage, gewissermaassen unterminirt (vergl. Al. Fig. 6-9). Die Spalte hat nun nebenstehende Form $\Upsilon$. Die Kehldeckelanlage stellt sich als eine halbmondförmige Kappe dar, die mit ihren beiden nach hinten gewendeten Seitenschenkeln die Falten, die den senkrechten Theil der Kehlkopfspalte begrenzen, umfasst. Von dem Vorderrande dieser halbmondförmigen Kappe ziehen noch deutlich erkennbar zwei kleine Leisten, die Reste der dritten Kiemenbogen, nach aussen längs dem hintern Rande des Zungengrundes hin (vergl. Fig. 8 und $9 \mathrm{~Kb}_{3}$ ).

Bei Embryonen von 15 N. L. und 16,5 S. S. Länge sind noch dieselben Dinge zu sehen, nur ist der Körper der Zunge wieder erheblich gewachsen und der ganze Zungenrïcken stärker nach oben gewölbt: die zweiten Schlundbogen mit ihrem medianen Vereinigungsstücke sind noch deutlich nach vorn durch eine Furche 
abgegrenzt, dabei sind dieselben ohne Weiteres als Anlage der Zungenwurzel kenntlich (vergl. Fig. 8, $\mathrm{Kb}_{2}=\mathrm{Zw}$.). Die Reste der dritten Schlundbogen sind noch unansehnlicher geworden. Die Mitte des hintern Randes der Kehldeckelanlage wächst hackenförmig über die den Kehlkopfeingang begrenzenden Falten hinweg und verdeckt so einen Theil der Längsspalte; bei noch weiterer Entwicklung (2,75 S. St.) wird der hintere Rand des Kehldeckels in der Mitte eingezogen.

Ueberwog schon bis hierher das Längenwachsthum der Zunge das Breitenwachsthum ganz erheblich, so gewinnt das erstere weiterhin einen ganz enormen Vorsprung. Immer ist es der eigentliche Zungenkörper, der daran den Löwenantheil hat. Die stark dorsalwärts gekrïmmte Zunge flacht sich mit der Ausgleichung des Sattelwinkels und der definitiven Gaumenbildung ab, doch geschieht dies in der Weise, dass der Zungenkörper sich im Winkel gegen den (vom zweiten Kiemenbogen stammenden) Zungengrund stellt, so dass dęr Flächenwinkel am Boden der Mundhöhle gewissermassen nach vorn riuckt.

Bei Embryonen von über $2 \mathrm{~cm}$ S. S. verlieren sich die Reste der dritten Schlundbogen, die noch in Fig. 9 als zwei quere Wuilste vom Vorderrande der Kehldeckelanlage nach auswärts ziehen, fast ganz; kaum dass an der entsprechenden Stelle noch ein kleiner Vorsprung am Rande des Kehldeckels zu sehen ist.

Es scheint für die iilteren Stadien zweckmässiger, nicht mehr die einzelnen Embryonen nach einander abzuhandeln, sondern gleich jedes Organ in seiner weitern Entwicklung durch die ganze Reihe der Embryonen, die grösser siud als $13 \mathrm{~mm} \mathrm{N.} \mathrm{L.,} \mathrm{hindurch} \mathrm{zu}$ verfolgen.

Bei Embryonen von $14 \mathrm{~mm} \mathrm{N.} \mathrm{L.} \mathrm{und} 15 \mathrm{~mm} \mathrm{S.} \mathrm{S.} \mathrm{existirt}$ noch die innere Mündung der zweiten Kiementasche in der Seitenwand der Mundhöhle und von ihr aus zieht sich durch 5-6 Schnitte von $1 / 30 \mathrm{~mm}$ Dicke ein Rest der Spalte selbst als ein enger Kanal in abnehmender Höhe nach hinten. Das Hautende der Kiemenspalte ist vollständig geschwunden. Bei Schweinsembryonen, die um $1 \mathrm{~mm}$ länger, waren die Reste der zweiten Kiemenspalte nur noch durch zwei Schnitte zu verfolgen; bei etwas grösseren ist auch diese letzte Spur vollkommen verschwunden.

Die dritte Kiemenspalte erhält sich als epithelialer Strang, der von der Schlundwand zur Haut zieht, bedeutend lïnger als die 
zweite. Noch bei Embryonen von 15 N. L. ist derselbe vollständig vorhanden. Bei Embryonen von 20 S. S. ist er unterbrochen, es existirt aber noch ein Epithelstrang, der von der Epidermis aus eine kleine Strecke in die Tiefe zieht, um dort blind zu endigen und ebenso geht von der Schlundwand ein schmaler Epithelstrang:

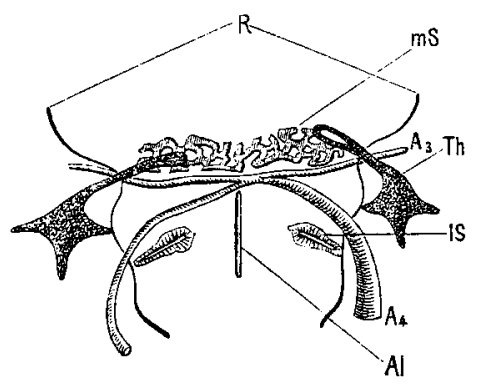

Fig. b.

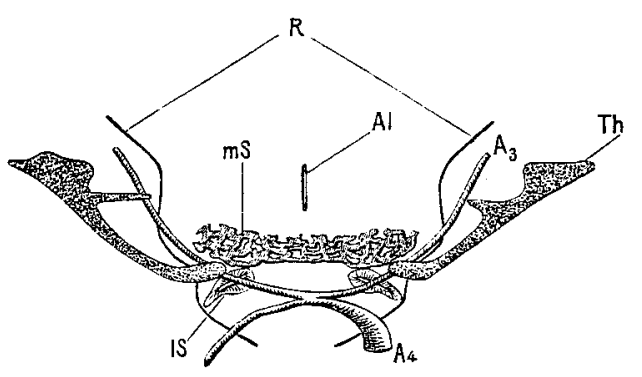

Fig. d.

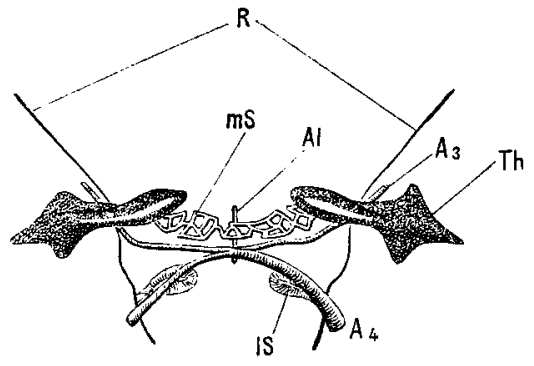

Fig. c.

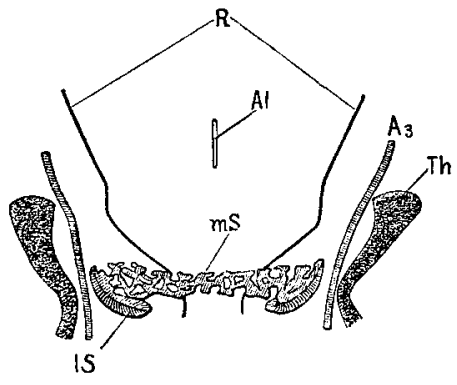

Fig. e.

Die Figuren b bis d sind Ansichten der Ventralfläche des Vorderdarms mit den derselben aufliegenden Drüsen und Gefässen, die nach Constructionen, wie sie $\mathrm{His}$ aus Querschnittserien herzustellen gelehrt hat, schematisirt gezeichnet wurden. Die Constructionen waren 40 fach vergrössert, die Figuren sind darnach um die Hälfte verkleinert. Vom Darm (R) sind nur die Randconturen wiedergegeben und innerhalb derselben der schlitzförmige Kehlkopfs-Eingang (Al). Die vordere, mediane Thyreoidea-Anlage ist in allen Figuren gleichmässig in Form eines schematischen Netzwerkes dargestellt, obgleich dieselbe bei Embryonen der Grösse von b u. c diese Ausbildung noch nicht erreicht hat. Aehnlich sind die laterale Schilddrüsenanlage (1S), die Thymus (Th) und die Gefässe $\left(A_{3}\right.$ u. $\left.A_{4}\right)$ in Bezug auf ihren Bau schematisirt. In welcher Weise die Verschmelzung der lateralen und der medialen Schilddrüsenanlage in d schematisirt ist, muss im Text nachgelesen werden. - b Embryo von $15 \mathrm{~mm}$ SS. - c Embryo von $16 \mathrm{~mm}$ SS. - d Embryo von $20 \mathrm{~mm}$ SS. - e Embryo von 22,5 SS. 
aus, der sich sehr bald zu einem massigen Epithellager verbreitert, mit dem immer noch die Thymus-Anlage zusammenhängt. Ueber die weitern Schicksale dieses Restes der dritten Kiemenspalte siehe unten. Die Veränderungen der für uns wichtigsten Gefässstämme, sowie der Anlagen der Thyreoidea und der Thymus bei Embryonen von $13 \mathrm{~mm}$ anfwärts sollen jetzt, um die Hanptpunkte dieser Vorgänge eindringlicher hervortreten zu lassen, in einer kurzen Uebersicht dargestellt werden; dann erst soll die specielle histologische Beschreibung der einzelnen Stadien folgen.

Wie oben sehon ausgefitibrt, verschiebt sich die vordere Thyreoidea-Anlage an der ventralen Seite der Kehlkopfspalte vorbei nach riickwärts, wobei zu bemerken, dass die Verschiebung desswegen noch grösser erscheint, als sie wirklich ist, weil die Kehlkoptspalte sich bei Embryonen von 13 bis $18 \mathrm{~mm}$ an hintern Rande merklich verkürzt (vergl. Holzschnitt b bis d). Der Verschiebung des quergestellten Epithelbandes, das dic vordere ThyreoideaAnlage darstellt, folgen bis.zu Embryonen von eirca $20 \mathrm{~mm}$ Länge stetig die dritten Aortenbogen, deren Ursprung aus der ventralen Aortenwurzel immer dicht am hintern Rande der Drỉsenanlage gefunden wird (Holzschnitt b bis $d \mathrm{~A}_{3}$ ). Diese dritten Aortenbogen werden bekanntlich zu den Art. earot. communes. Aus der Vereinigungsstelle derselben entspringen auch die nach hinten ziehenden vierten Aortenbogen, von denen der linke bald viel stärker erscheint und die directe Fortsetznng der ventralen Aorten wurzel, den bleibenden Aortenbogen, bildet, während der rechte zur Art. subclavia derselben Seite wird (Holzschnitt $A_{4}$ ). Bei Embryonen von über $20 \mathrm{~mm} \mathrm{S.} \mathrm{S.}$ rïcken die Thyreoidea-Anlage und die Vereinigungsstelle der Carotides communes auseinander, indem die letztere sich in einem rascheren Tempo nach rückwärts verschiebt, als die erste. Diese Differenz wird mit vorschreitendem Wachsthum immer erheblicher (vergl. Holzschnitt e $A_{3}$ ). Diejenigen Stadien, in denen sich beim Schweine ein besonderer Ursprungsstamm für beide Carotiden und die rechte Subclavia, die Armkopfarterie, herausbildet, habe ich nicht verfolgt. Die paarigen Anlagen der Thymus hängen bei Embryonen von $13 \mathrm{~mm}$ durch den Rest der dritten Kiemenspalte mit der seitlichen Schlundwand zur Seite des vordern Endes des Kehlkopfeinganges zusammen (vergl. Th. Holzschnitt b). Auch nach Lösung dieser Verbindung behält der Anfang des Thymusschlauches seine Lage zu beiden Seiten des Kehlhopfschlitzes bei 
(vergl. Holzschnitt $d$ Th.). Dieser dorsale Anfang des Thymusschlauches wird von dem mächtigen Epithellager gebildet, das, wie ich oben beschrieben habe, als ein Rest der obliterirten dritten Schlundspalte aufzufassen ist. Dasselbe liegt gerade ventralwärts vor der Theilungsstelle der Carotis communis. Der Thymussehlauch selbst zieht von diesem dorsalen Anfange aus bei Embryonen von 13 N. L. ventralwärts und zugleich nach innen und vorn (vergl. Holzschnitt b Th.), um sich mit seinem blinden, hackenförmig nach innen umgebogenen Ende dicht am Pericard neben der Austrittsstelle der Aorta aus demselben zu lagern. Der Thymusschlauch geht dabei dicht an der hintern Seite der dritten Aortenbogen (carotides communes) vorbei; an die vordere Seite der letztern grenzt, wie gesagt, die Thyreoida-Anlage an (vergl. dazu Holzschnitt b). Mit der Rückwärtsverschiebung der Vereinigungsstelle der Carotiden und des ganzen vordern Herzendes wandert auch das Ende der Thymus nach hinten. Da der dorsale Anfang derselben aber an Ort und Stelle bleibt, muss der epitheliale Schlauch immer länger werden. Während die Längsaxe desselben Anfangs von der dorsalen Seite nach vorn und ventralwärts gerichtet war, geht derselbe jetzt erst schräg nach hinten und ventralwärts, um schliesslich beinahe rein nach hinten gerichtet vor den grossen Halsgefässen in die Brustapertur herabzuziehen (vergl Holzschnitt b-e Th). Wenn man will, beschreibt demnach während des Wachsthums vom Stadium 13 NL. an das ventrale Thymusende um das dorsale einen Bogen nach hinten, nur ist dazu noch zu merken, dass der ganze Schlauch dabei um ein Vielfaches in die Länge wächst.

Nun blieben noch die drüsenartigen, ventralen Ausstülpungen der seitlichen Schlundwand zu Seiten des Kehlkopfschlitzes, die ich oben als Reste der vierten Kiemenspalte gedeutet babe, za besprechen; dieselben liegen bei Embryonen von $13 \mathrm{~N}$. L. hinter den vierten Aortenbogen (vergl. Holzschnitt $b-$ IS.). In den folgenden Stadien lösen sich dieselben mit ihrem verdünnten dorsalen Ende von ihrem Mutterboden $a b$ und verschieben sich auch ein wenig nach hinten, so dass dieselben nun hinter dem Keblkopfscblitze zur Seite des Anfangs der Trachea gefunden werden (vergl. Holzschnitt c-e 1S.). Doch ist diese Verschiebung ungleich geringer, als die der übrigen besprochenen Gebilde, und man kann leicht constatiren, wie zuerst die vierten Aortenbogen, dann die ventralen Enden der ThymusAnlagen und schliesslich die dritten Aortenbogen an der ventralen 
Seite dieser Gebilde vorbei nach rückwärts wandern (vergl. Holzschnitt $\mathbf{b}$ bis $\mathbf{e})$. Die vordere Thyreoidea-Anlage aber rückt während dieser Veränderung den zu besprechenden paarigen Gebilden immer näher und kommt schliesslich, da sie etwa in derselben Ebene mit denselben liegt, mit ihnen in Berührung. Es geschieht dies meist bei Embryonen von $19 \mathrm{~mm} \mathrm{S.} \mathrm{S.} \mathrm{(vergl.} \mathrm{Holzschnitt} \mathrm{d,} \mathrm{wo} \mathrm{die} \mathrm{Be-}$ riihrung eben erreicht ist). Nun bemerkt man eine Erscheinung, die mich anfänglich so sehr überraschte, dass ich eine grosse Zahl Schnittserien von Embryonen von 18-22 mm Länge anfertigte, um mich vor jeder Täuschung sicher zu stellen. Bei Embryonen von etwas über $20 \mathrm{~mm}$ S. S. verschnelzen nämlich die Epithelbalken der unpaaren Thyreoidea-Anlagen seitlich mit den blinden ventralen und zugleich etwas nach innen gerichteten, erweiterten Enden dieser paarigen Gebilde. Bei wenig älteren Embryonen werden die letzteren, die sich gleich nach der Verschmelzung an dem verschiedenen histologischen Bau noch deutlich erkennen lassen, in ein Epithelbalkennetz von demselben Aussehen umgewandelt, wie das der unparen Anlage. Es ist also ohne Zweifel, dass der epitheliale Theil der Thyreoidea durch Verschmelzung zweier ursprïnglich räumlich getrennter und histologisch versehieden gebauter Bestandtheile hergestellt wird; der eine von diesen, der unpaare, wächst in der Medianlinie aus dem Epithel der Vereinigungsstelle der zweiten Kiemenbogen aus, derselbe nimmt frühzeitig die bekannte Netzstructur an, der andre paarige Theil wird durch zwei schlauchartige, ventrale Aussülpungen der vierten Kiemenspalten, die etwas nach innen convergiren, dargestellt; letztere verlieren erst nach der Verschmelzung mit dem medianen Antheil, der sich allmählich nach hinten verschiebt, ihre einfache Schlauchform und wandeln sich in ein Netz von Zellbalken um.

Es ist klar, dass von den letzteren die seitlichen Lappen der Schilddrüse geliefert werden. Beim Schwein schliessen sich dieselben später sehr dicht vor der Luftröhre aneinander. Ich werde die von den vierten Kiemenspalten abstammenden Schläuche von nun an kurzweg als seitliche oder hintere Thyreoidea-Anlagen bezeichnen. Die ansführliche histologische Beschreibung derselben muss natürlich mit der Beschreibung der unpaaren Anlage für jedes Stadium zusanmen geschehen. 


\section{Specielle histologische Beschreibung der Thyreoidea- und Thymus-Anlagen.}

Die vordere Thyreoidea-Anlage stellt bei Embryonen von $15 \mathrm{~mm}$ S. S. und $14 \mathrm{~N}$. L. ein vor dem Kehlkopfschlitze tief im Boden der Mundhöble gelagertes, queres Epithelband dar, dessen seitliche verdickte Enden nur ganz unbedeutend dorsalwärts, etwas merklicher nach hinten umgebogen sind. Von dem kernreichen embryonalen Bindegewebe der Umgebung hat sich eine dünne Schicht Faserzellen der Oberfläche des Epithelbandes parallel stratificirt, und bildet eine Art Kapsel um dieselbe; hie und da weichen die Spindelzellen anf den Schnitten auseinander und umsehliessen Spalten, von denen es schwierig zu unterscheiden ist, ob dieselben schon mit Blutgefässen zu identificiren sind, oder nicht. Die Oberfläche des Epithelbandes selbst ist mit mannigfachen Vorsprüngen, zwischen denen das Bindegewebe der Umgebung eindringt, unregelmässig besetzt; in den dickeren seitlichen Enden bemerkt man schon Andeutungen einer netzförmigen Anordnung der Zellstränge, wie sie bald viel deutlicher hervortritt. Die äusserste Zellschicht in den Zellbalken bildet iiberall eine Lage regelnässig nebeneinander gestellter, hoher Cylinderzellen mit verlängerten Kernen. Zwischen den Cylinderzellenlagen findet sich an meinen Präparaten eine körnig-fädliche Substanz mit runden Kernen, in der sich nur selten deutliche Zellgrenzen erkennen lassen. Lumina, wie sie sich zur selben Zeit in den benachbarten Enden der Thymus-Anlage deutlich zeigen, sind in der vorderen Thyreoidea-Anlage nur hie und da zu erkennen. Bei etwas jüngeren Embryonen (14 N. L.) sind dieselben häufiger und man bekommt den Eindruck, als handle es sich vielleicht um einen eng hin- und hergebogenen und zusammengeknäuelten Schlauch.

Die viel weiter rückwärts, in der Querlinie des hintern Endes der Kehlkopfspalte, gelegenen seitlichen Thyreoidea-Anlagen baben zur selben Zeit das Aussehen einfacher, schlauchförmiger Drüsen, die mit verschmälertem Halse in die Seitenwand des Schlundes einmiinden und im Bogen um die Kehlkopfspalte ventralwärts herumziehen. Die verdickten Enden derselben reichen etwas weiter ventralwärts, als der ventrale Rand des Kehl- 
kopfes. In der ganzen Länge des Schlauches siebt man ein deutliches Lumen; die Ränder desselben sind meist glatt; die Epithelbekleidung ist mehrschichtig, am dicksten an den ventralen Enden. Zunächst dem Lumen erscheint eine streifige, kernfreie Grenzschicht.

Bei Embryonen von $16 \mathrm{~S}$. S. hat sich trotz der merklichen Verschiebung der Drtisenanlagen (vergl. Holzschnitt c mit b), die histologische Ausbildung kaum verändert.

Bei Embryonen von $18 \mathrm{~mm}$ S. S. (vergl. Fig. $11 \mathrm{~m} \mathrm{~S}$.) ist die mediane Thyreoidea-Anlage schon vollkommen in ein Netzwerk von Zellbalken aufgelöst, in dessen Maschen blutgefüllte Capillaren leicht erkennbar sind, wie dies von W. Mill er in seiner ausgezeichneten Arbeit beschrieben und abgebildet worden ist. In einer Beziehung erinnert das Bild noch an den früheren Zustand, nämlich darin, dass zwei tibereinanderliegende Querzüge von Zellsträngen besonders scharf hervortreten, die freilich durch reichliche dorso-ventrale Verbindungsbalken zusammenhängen. Die einzelnen Zellbalken bestehen aus einem peripheren, ziemlich regelmässigen Cylinderzelllager und zwischen den Enden dieser in wechselnder Menge eingeschobenen, runden Kernen mit undeutlich geschiedenem Protoplasma. Um die Zellbalken differencirt sich eine Spindelzellschicht, deren Kerne von denen der Kapillaren stellenweise kaum zu unterscheiden sind (vergl. Fig. 11).

Der Hals, mit dem die schlauchförmige, seitliche ThyreoideaAnlage am Schlunde befestigt ist, erscheint jetzt sehr verdünnt, doch ist in demselben meist ein noch ganz deutliches Lumen erkennbar (vergl. Figur 12 IS). Dieser dünne Hals treibt eine solide, geknickte Zellsprosse nach hinten. Das kolbenförmige, ventrale Ende hat seine Wandungen verdickt, erscheint aber im Uebrigen nicht verändert. Bei Embryonen von $19-20 \mathrm{~mm}$ S. S. hat sich das Zellbalkennetz der medianen ThyreoideaAnlage dem Grunde der Schläuche, die die seitlichen Thyreoidea-Anlagen darstellen, bis zur Berïhrung genähert (vergl. Holzschnitt d). Letztere haben sich vom Schlunde losgelöst. Im Inneru derselben existirt immer noch ein Lumen, obgleich bei der starken Dickenzunahme der dasselbe begrenzenden Zellschichten gute Schnitte zur Wahrnehmung desselben gehören. Die ganze Anlage hat jetzt ausgeprägte Kolbenform. Der verdïnnte Stiel des Kolbens strebt dorsalwärts gegen die seitliche Schlundwand, ohne dieselbe zu erreichen. Das verdickte ventrale Ende zeigt keine 
regelmässig abgerundete Schnittcontur mehr, sondern erscheint hie und da ansgebuchtet. An einem Exemplar derselben Tracht waren schon einzelne Stränge der medianen Schilddrüsen-Anlage mit dem vordern Ende der seitlichen Schläuche in sehr deutliche Verbindung getreten. Bei Embryonen von circa $21 \mathrm{~mm}$ S. S. tritt die vollkommene Verbindung der medianen Thyreoidea-Anlage mit den seitlichen ein, und zwar geschieht dieselbe zuerst an den Enden und den ventralen Flächen der letzteren. Holzschnitt e giebt die Art und Weise der Verbindung nicht ganz genau wieder; es misste die mediane Anlage die ventralen Enden der paarigen iberdecken und iiber dieselben seitlich hinausragen. $\mathrm{Da}$ aber so die Verschmelzung selbst in der Zeichnung sich nicht hätte andeuten lassen, zog ich es vor, die beiden Anlagen im Bilde nebeneinander, statt aufeinander zeichnen zu lassen. Die verdünnten dorsalen Theile der paarigen Anlagen ziehen noch frei nach aussen, dorsalwärts und nach vorn (vergl. Fig. 13 und 14 lS.); dieselben zeigen bäufig ein dentliches Lumen, während ein solches in dem verdickten medialen Theile, der die Verschmelzung eingeht, nicht mehr zu erkennen ist, - nur einzelne unregelmässige Lücken sah ich noch in der dichtkernigen Masse desselben. Zur selben Zeit wird die Oberfläche des ventralen Kolbenendes unregelmässig. Die Schnittcontur desselben zeigt mannigfache Vorsprünge und Einbuchtungen, in die Capillaren eindringen (vergl. Fig. 13 und 14); es bedeutet dies den Beginn der Auflösung der seitlichen Thyreoidea-Anlage in ein eben solcbes Zellbalkennetz, wie das war, aus dem die mediane Anlage schon vor der Verschmelzung bestand. Doch selbst, wenn die Verbindungen der medianen Anlage mit den paarigeu schon recht zahlreich und dicht geworden sind, sieht man an Präparaten, die in M ïller'scher Flüssigkeit und Alcobol gehärtet und mit Alaun-Cochenille gefärbt sind, die Zusammensetzung der Drüse aus ungleichartigen Bestandtbeilen, einem mittleren und zwei seitlichen paarigen, sofort an der Färbung. Die von den seitlichen Thyreoidea-Anlagen abstammenden Theile zeigen sich viel intensiver roth gefärbt, als das Zellbalkennetz der medianen Anlage. (So in Fig. 13, in Fig. 14 war der Unterschied viel geringer und ist demgemäss auch in der Zeichnung nicht angedeutet.) Es rührt dies, so viel ich sehen kann, nicht bloss von der sicher vorhandenen dichteren Lagerung der Kerne, sondern auch von einer stärkern Aufnahme des Färbungsmittels seitens der Zellen und Kerne der 
paarigen Anlagen her. An den Verschmelzungsstellen selbst gehen die Zellbalken der medianen Anlagen unmerklich in die Substanz der paarigen iiber, die sich hie und da in Vorspriingen den Balken der ersteren entgegen erhebt (vergl. Fig. 13 und 14).

$\mathrm{Zn}$ bemerken ist noch, dass die Zellbalken der medianen Anlage nicht mehr vorwiegend quer zusammenhängen, sondern jetzt so von Blutgefässen durchbrochen sind, dass die dorsoventralen Züge um meisten in die Augen fallen. - Da die Dimensionen bei Embryonen bekanntlich niemals der Entwickelungsböhe genau parallel zunehmen, so ist es vielleicht zweckmässig, dieses wichtige Stadium, in dem die Verschmelzung der medianen mit den paarigen Thyreoidea-Anlagen eben eingetreten ist, durch die Angabe näher zu bezeichnen, dass in demselben die Anlagen der Keblkopfknorpel als Verdichtungen der Bindesubstanz sich nur erst mit Mühe wahrnehmen lassen; sobald dieselben deutlich hyalinknorplig geworden sind, ist die Verschmelzung der diskreten Bestandtheile vollkommen geworden und die in Zellbalken aufgelösten seitlichen Anlagen kaum mehr von der medianen zu unterscheiden. - Wichtig für die Wahrnehmung dieser Verhältnisse ist auch die Scbnittrichtung; dieselbe muss mit der Ebene, in der die drei Bestandtheile der Driisenanlage enthalten sind, einigermassen zusammenfallen, damit man dieselben auch in einem Schnitte nebeneinander sieht. Es geniigte die in der Einleitung beschriebene Schnittrichtung dieser Anforderung für meine Objecte ziemlich vollkommen. Auffällig war die Verschiedenheit der Massenentwicklung des paarigen und der unpaaren Componenten der Driise bei Schnittserien durch verschiedene Embryonen ein und derselben Tracht. Ich vermag nicht zu entscheiden, ob dies auf Rechnung einerverschiedenen Entwickelungshöhe oder auf individuelle Unterschiede, die dann auf die bekannten Varianten der Schilddrüse bezogen werden könnten, zu setzen ist.

Bei Embryonen von $26 \mathrm{~mm}$ S. S., bei denen die Driisenanlage schon einen einheitlichen, mit den Enden dorsalwärts und nach vorn aufgebogenen Drïsenkörper darstellt, dessen Mittelstück an dem Anfang der Trachea gelègen ist, erkennt man die besondere Abstammung der seitlichen Theile des Zellbalkennetzes noch an den engeren Maschen und den dickeren Balken derselben. An einzelnen Stellen finden sich noch compacte Anhäufungen epithelialer Zellen, in die noch keine Blutgefässschlingen eingedrungen sind. Bei einem Embryo von 37 mm war die Drüse zu 
einem von einer glatten Bindegewebskapsel umschlossenen Körper von ovalem Querschnitt geworden, der vor dem Anfangstheil der Trachea gelegen war und nur durch einen tief ins Innere ein-schneidenden Bindegewebszug die Entstehung aus zwei aneinandergrepressten, vorher in die Quere ausgezogenen Seitentheilen erkennen liess. Es lassen sich keine histologischen Unterschiede zwischen der Mitte und den Seitentheilen mehr erkennen, die Zellbalken der Drïse scheinen im Beginn der Abschnïrung zu kugligen Blasen zu stehen. Eine specielle Aufmerksamkeit habe ich den weiteren Entwickelungsstadien der Driise nicht gewidmet.

Bei Embryonen bis zu $18 \mathrm{~mm}$ S. S. erhält sich als Rest der dritten Kiemenspalte ein epithelialer Strang, der von der seitlichen Schlundwand bis zur Epidermis reicht. Derselbe ist noch eine Strecke weit mit einem dentlichen Lumen versehen. Doch blieb ich im Ungewissen, ob dasselbe noch mit der Schlundlichtung communicire, jedenfalls reicht der Spalt in demselben sehr nahe an die letztere heran; das Stück zunächst der äussérn Haut ist ganz bestimmt solid. Gleich nach dem Abgange vom Schlunde durchsetzt das Rohr in einer gebrochenen Linie ein grosses Epithellager von dreieckiger oder viereckiger Form und zwar nahe am dorsalen Rande desselben. Dieses Epithellager stellt, wie oben erwähnt, anch einen Rest des friiber dorsalwärts ausgebnchteten dritten Kiemenspaltes dar. Von dem untern, spitz ausgezogenen Ende dieses Epithellagers geht der epitheliale Strang der Thymus-Anlage ans, über dessen Verschiebung und Verlängerung wir oben ausführlich berichtet haben. Derselbe zeigt in seiner ganzen Länge ein deutliches Lumen, umgeben von einem schönen, mehrschichtigen Epithel (vergl. Fig. 11 Th.); doch communicirt dasselbe mit dem Lumen des an obern Rande des Epithellagers gelegenen Restes der dritten Kiemenspalte nicht. Bei Embryonen von etwas ïber $20 \mathrm{~mm}$ S. S. sind dieSchlund- und die Hautverbindungen des Restes der dritten Kiemenspalte geschwunden, nur ein zipfelförmig ausgezogener, ibrigens noch hohler, lateraler Fortsatz des Epithellagers erinnert an die frühere Hautverbindung. Das Epithellager hat eher noch an Ausdehnung gewonnen. Der aus seinem untern Ende hervorgehende Thymusstrang lässt im dorsalen Abschnitt kein deutliches Lumen mehr erkennen, erst gegen das unterste, medialwärts abgebogene Ende desselben, das dicht am Pericard zur Seite der Austrittsstelle der Aorta aus demselben liegt, zeigt der Strang noch eine deut- 
liche Lichtung. Dieses ventrale Ende der Thymus-Anlage erscheint unregelmässig conturirt, es ist im Anfang schwer zu unterscheiden, ob die Vorsprünge der Contur auf Sprossenbildung des Epithellagers oder auf eine Aufknäuelung desselben zurïckzuführen sind.

Bei Embryonen von $25 \mathrm{~S}$. S. ist das Epithellager, welches das vordere Ende der Thymusanlage darstellt, noch sicher neben dem Kehlkopf zu erkennen; an dem Herzende sieht man jetzt deutlich, dass das anfangs einfache Rohr seitliche, solide Aeste zu treiben anfängt, ganz nach der Art, wie sich sonst eine Drüsenanlage verzweigt.

Bei einem Embryo von $35 \mathrm{~mm}$ S. S. hat der ganze, am Halse vor den grossen Blutgefässen herabziehende Strang der epithelialen Thymus-Anlage seitliche Sprossen getrieben, das vordere Epithellager dagegen hat sich verloren und der Strang endet vorn zugespitzt an der Aussenseite der Cartilago Thyreoidea etwa auf Querschnitten, die den untern Rand der Giessbeckenknorpel treffen. Merkwürdigerweise nimmt die Sprossenbildung gegen das Herzende zu wieder ab, so dass die Thymus-Anlagen als zwei feine, dicht aneinander gelagerte Epithelröhren dorsal hinter dem Manubrium sterni wegziehen, um dann in einen mächtig verzweigten Epithelbaum, das eigentliche Herzende, einzumuinden. Jetzt ist in der ganzen Anlage nirgends mehr ein Lumen zu erkennen.

Die weitere Entwicklnng der Thymus habe ich nicht verfolgt.

Der Vergleich der auf vorstehenden Seiten niedergelegten Darstellung der Entwicklung der Thyreoidea mit den Angaben Wöltler's zeigt, dass ich seine Schilderung nur in einem Puncte bestätigen kann, nämlich in dem Bilde, das er von der Beschaffenheit der zweiten (W.'s erster) Kiementasche bei Schweinsembryonen von $9 \mathrm{~mm}$ Länge entwirft und das er auf p. 17 mit einer zweckmässigen, schematischen Figur erläutert hat. Nur finde ich die dorsale Fortsetzung der Kienentasche (c. bei W.) nicht rinnenartig gegen die Mundhöhle geöffnet, sonderu blindsackförmig ausgebuchtet. Durchaus bestreiten muss ich aber die AnsichtW.'s, dass das Sagittalschnittbild des medialen Endes der rinnenartigen ventralen Fortsetzung: der Kiemenspalte im Boden der Mundhöhle die Grundlage fur die Behauptung der Autoren Remak, Müller, Sessel, Kölliker u. A. m., dass die Thyreoidea aus einer medianen Ausbuchtung 
der ventralen Schlundwand entstehe, abgegeben habe; ein Querschnitt, wie der in Fig. 10 abgebildete, bätte Wölfler überzeugt, dass zwischen jenen rinnenartigen Fortsetzungen der zweiten Kiemenspalten eine an der Medianebene gelegene, gesonderte ThyreoideaAnlage existirt, die durchaus nicht von jenen berzuleiten ist. Die Stütze, die W. seiner Anschaunng dureh den Bezug auf die Figuren Moldenhauer's (Nr. VII p. 125) zu geben sucht, fällt in sich selbst zusammen, da bei dem zweitägigen Hiihnerembryo, dem die eine Moldenha uer'sche Serie entnommen ist, überhaupt erst eine, und zwar die erste Schlundtasche, die tubotympanische, gebildet war. Die seitliche Ausstülpung, die diese darstellt, hat gar nichts mit der weiter nach hinten folsenden Vertiefung im Boden des Vorderdarms, aus der sich nach Moldenhauer. Thyreoidea und Lunge hervorbilden sollen, zu thun. Die zweite Serie Moldenhauer's (1. c. Taf. VII Fig. 11-19) enthält wieder nur die erste, die tubotympanische Schlundtasche, und zu allem Ueberfluss von dieser vollkommen getrennt, in Fig. 18 und 19, in denen die Schlundtasche gar nicht mehr sichtbar ist, eine kleine, mediane, sackförmige Ausstïlpung in den Boden der Mundhöhle, - die vordere Anlage der Thyreoidea.

Dass aber diejenige Schlundspalte, welche die von Moldenhauer beschriebene Form hat, und die er selbst (vergl. seine Fig. 1) hinter das Ohrbläschen verlegt, die zweite und nicht die erste ist, darauf habe ich schon oben (Seite $272 \mathrm{Anm} .1$ ) ausdricklich hingewiesen. Aus der ausführlichen Darstellung meiner Befunde ergiebt sich aber, dass ich auch dém Epithel der zweiten Schlundspalte keine Betheiligung am Aufbau der Thyreoidea-Anlage zugestehen kann. Die zweite Kiemenspalte vergeht vollkommen, obne Spuren zuriickzulassen. Die Hauptursacbe, die W. zu einer so irrigen Anschauung verfïhrt hat, scheint mir in der Wahl der Schnittrichtung zu liegen. Jeder, der sich mit solchen Ohjecten selbst arbeitend beschäftigt hat, weiss, wie äusserst schwierig die Orientirung an Sagittalschnittserien ist. Ich glaube, ich kann nach dem Gesagten darauf verzichten, eine entsprechende Umdeutung jeder einzelnen Figur W.'s vorzunehmen, theilweise ist mir eine solche auch ganz unmöglich; nur einiges will ich besonders hervorheben. Die mit Auswüchsen bedeckte Schnittfigur der zweiten Kiemenspalte (Taf. II Fig. 9 und 12 W's 1 Sp.) braucht durchaus nicht auf eine Proliferation des Epithels derselben bezogen zu werden, sondern erklärt 
sich wohl daraus, dass die Schlundtasche eine sehr complicirte Form mit mannigfachen, oben beschriebenen Ausbuchtungen und Vorsprïngen besitzt und daher in einem sie ganz schräg treffenden Schnitte keine einfache Schnittcontur aufweisen kann, ja mitunter mehrfach angeschnitten werden muss. Die specielle Auflösung jeder einzelnen Schnittfigur halte ich, nachdem ich die Form der Schlundspalte plastisch dargestellt habe, nicht für nöthig, übrigens, wie gesagt, auch für recht schwierig. Fig. $17 \mathrm{~W}$.'s soll das Kiemenspaltenepithel in Proliferation zeigen. Ich kann dieser gegenüber nur sagen, dass die Epithelstränge in derselben mir merkwürdig mangelhaft begrenzt erscheinen; während die Schlundspalte selbst scharfe Ränder zeigt, so dass der Verdacht nahe liegt, es handle sich hier um eine falsche Auffassung eines vielleicht in der Färbung nicht scharf genug differenzirten Präparates, so dass an Stellen, wo Epithel- und Bindesubstanzgrenzen schräg zur Schnittfläche anstehen, die Grenze übersehen ist. Es ist bekannt, dass bei Säugethierembryonen die Abgrenzung vom Epithel und dem kernreichen Bindegewebe mitunter bedeutende Schwierigkeiten macht und nur bei scharfen Tinctionen gelingt. Ueber die weitere Ausbildung der Schilddrtisenanlage, über die Netzform der Zellbalken hinaus, babe ich keine Untersuchungen angestellt und habe daher auch keine Veranlassung, auf die W.'sche Darstellung derselben hier näher einzugehen.

Stieda hat sich das grosse Verdiensterworben, den Zusammenhang der Schilddrüssen-Anlage mit $z$ wei von einerder letzten Kiemenspalten jeder Seite ausgehenden drïsenförmigen Ausstîlpungen, die den Anfang der Trachea oder das Ende des Kehlkopfes umfassen, richtig erkannt zu haben. Ihm ist nur entgangen, dass dicselben sich mit einer schon früher vorhandenen medianen Anlage secundär verbinden, obgleich auch ihm (vergl. IV p. 17 Zeile 10-14) der Mangel der Verbindung der beiden seitlichen Anlagen der Schilddrüse mit dem Mittelstïck an seinen Querschnitten durch Schweinsembryonen anfgefallen war. Die Verbindung, die er bei offenbar für die Entscheidung dieses Punctes zu alten Schafen auffand (cf. Fig. 10), verleitete ihn zu der Annahme, „dass das Mittelstick sehr früh aus den seitlichen Anlagen hervorwächst." Auch ist es dem Autor nicht gelungen, die Kiemenspalte zu bestimmen, von der die Ausstülpung ausgeht. Irrthümlicherweise 
lässt er die Thyreoidea von derselhen Kiemenspalte, wie die Thymus auswachsen. Meine Schnittbilder stimmen in rollkommen genügender Weise mit denen Stieda's überein, nur in einzelnen untergeordneten Puncten finden sich Differenzen. In Fig. 3 Taf 1, die eine vollkommen richtige Darstellung der kolben- oder birnförmigen paarigen hintern Schilddrüsen-Anlagen giebt, ist die Contur derselben mit einer grossen Anzahl unregelmässiger Vorsprünge besetzt, trotzdem steht die Anlage noch mit dem Epithel der Schlundspalte in Zusammenhang; ich fand die Anlagen, wenn ihre Contur so stark unregelmässig wurde, meist schon abgelöst, doch können hierin leicht individuelle Verschiedenheiten vorkommen. Fig. 6 Taf. 2 von einem $6 \mathrm{~mm}$ langen Schafembryo zeigt die birnförmigen, hohlen, hintern Thyreoidea-Anlagen genau so, wie ich sie bei Schweinsembryonen beschrieben habe, nur sind dieselben nicht, wie St i ed a glaubt, secundäre Ausstiilpungen der Thymussäckchen, sondern entstehen selbständig hinter denselben, wahrscheinlich von Resten der vierten Kiemenspalten. Fig. 10 Taf. 2, Schafembryo von $14 \mathrm{~mm}$ Länge, zeigt, wie schon oben erwähnt, die unpaaren Thyreoidea-Anlagen in Verschmelzung mit der medianen Anlage begriffen. Zufällig hängen anf diesem Schnitte die Seitentheile der letzteren in der Mitte nicht zusammen, ein Umstand, der vielleicht Stieda in der Annahme, dass die mittleren Theile aus den seitlichen hervorwachsen, bestärkt hat. Auch konnte ich bestätigen, dass das dorsale Ende der seitlichen Thyreoidea-Anlage in diesem Entwickelungsstadium noch einen Gang mit einem deutlichen Lumen darstellt. Eine epitheliale Anlage der Carotidendrüse, wie sie St. abbildet und beschreibt, habe ich nicht finden können. An dem dorsalen Ende der paarigen Thyreoidea-Anlagen fand ich, wie oben bemerkt, oft einen seitlich abgebogenen Epithelfortsatz (vergl. Fig. 13 und 14), der genau der Stieda'schen Anlage der Carotidendrüse (vergl. bei diesem Taf. 2 Fig. 10 gl. c.) entspricht, vermochte aber keine Weiterentwicklung desselben wabrzunehmen; ich bin eher geneigt, falls derselbe sich mitunter abschnüren und vergrössern sollte, die dorsalen, accessorischen Schilddrisen von demselben herzuleiten. Fig. 11 entspricht meiner Fig. 14. In Bezug auf die netzförmige Auflösung der primären epithelialen Schilddrüsen-Anlage muss ich mich entgegen Kölliker der Darstellung W. Müller's anschliessen. Auch $\mathrm{His}$ hat die paarigen Anlagen in der Thyreoidea gesehen, aber, wie es scheint, mit auf die Thymus bezogen. Der untere (meiner 
Nomenclatur nach hintere) Epithelstrang, den er I p. 56 bescbreibt, der nach auswärts vom fïnften Aortenbogen liegt, ist dieser seiner Lage nach identisch mit der seitlichen Thyreoidea-Anlage. His bemerkt auch noch dazu: Diese auf dem Durchschnitt geschlossen aussehenden Epithelfiguren scheinen iubrigens die blinden Endbuchten der dritten und vierten Schlundspalten zu sein. Das unter b p. 882 (VIII) von K ölliker bei einem 14 tägigen Kaninchenembryo beschriebene, thymusähnliche Organ an der ventralen Seite der noch sehr unentwickelten Thyreoidea dicht am vordersten Arcus aortae von $0,13 \mathrm{~mm}$ Länge, $54 \mu$ Dicke" ist sehr wahrscheinlich auf die seitlichen Schilddrüsen-Anlagen zu beziehen. Kölliker setzt noch hinzu: "Dieses Organ lag so dicht an den Schilddrtisen an, dass ich dasselbe zuerst für einen Theil desselben hielt, wogegen starke Vergrösserungen leicht den verschiedenen Bau nachwiesen." Diese Verschiedenheit des Baues der paarigen Schilddriisen-Anlagen von dem der mittleren ist aber, wie oben zu lesen, in der That anfangs vorhanden. In wieweit die ailteren Angaben Huschke's, der die Schilddrüse als paarige Anlagen vom ersten Kiemenbogen ableitet, und die von Stieda citirten A r nold'schen Angaben Beziehungen zu den von Stieda gefundenen paarigen Anlagen, die ich auf die vierten Kiemenspalten zurückführe, besitzen, vermag ich nicht zu entscheiden. - Jedenfalls scheint es allen bisherigen Autoren entgangen zu sein, dass die Schilddruse durch Verschmelzung aus zwei ursprünglich unähnlichen, getrennten Bestandtheilen, einem unpaaren und einem paarigen, entsteht, wie ich dies oben ausführlich dargelegt habe. Dass die von mir gefundene Entwickelungsweise auch für das Schaf gilt, beweisen die Untersuchungen Stieda's, der die seitlichen Anlagen der Drise auch bei diesen auffand. Aus einer $\mathrm{H}$ is s'schen Notiz geht es, wie oben bemerkt, mit grosser Wahrscheinlichkeit hervor, dass dieselben Anlagen auch beim menschlichen Embryo vorbanden sind. Ich bin fest ïberzengt, dass die Bildungsweise der Drüse bei allen Säugethieren die gleiche ist und habe dieser Ueberzeugung dadurch Ausdruck gegeben, dass ich im Titel nicht „Die Derivate der embryonalen Schlundbogen und Schlundspalten von sus scropha" sondern „der Säligethiere" schrieb, obgleich meine eigenen Untersuchungen sich nur auf Embryonen jener einen Art erstreckten.

Für alle übrigen Wirbelthierklassen ist durch die ausgezeichnete Arbeit W. Müller's (XI) das Vorhandensein einer medianen 
Schilddrüsen-Anlage mit Sicherheit nachgewiesen; - in wie weit auch die paarigen Anlagen vorkommen und welche Einflitsse deren Vorhandensein oder Feblen auf die Form der Drïse bei den einzelnen Klassen hat, das bedarf einer erneuten Untersuchung, zu der mir, hoffe ich, das nächste Frühjahr Musse und Material bringen wird. Dann denke ich auch an Säugethierembryonen aus andern Familien, namentlich an den leicht in vollständiger Serie zu erreichenden Kaninchenembryonen ergänzende Untersuchungen anstellen zu können. Bis dahin will ich mich jeder vergleichenden und speculativen Betrachtung über die immerhin merkwürdige Bildungsweise der Schilddrüse enthalten und versage mir auch, so verlockend es erscheint und so sehr Herr Professor Hasse in der Discussion dazu anregte, eine Erklärung der bekannten Varietäten der Schilddrüse, sowie des Vorkommens von Nebenschilddrüsen auf meine Befunde zu gründen.

Kölliker (Nr. VIII p. 876 u. folg.) hat bekanntlich zuerst anf den epithelialen Bau der embryonalen Thymusanlagen der Säugethiere aufmerksam gemacht, und ausserdem auch erkannt, dass diese Gebilde unzweifelhaft umgewandelte Kiemenspalten sind, nur blieb er ungewiss, ,welche von den bintern Kiemenspalten, die zweiten, dritten oder vierten" zur echten Thymus sich umbilden. His hat die Kölliker'sche Angabe bestätigt und Stieda den directen Zusammenhang des Thymusschlauches nit einem Kiemenspaltenreste nachgewiesen. Ich konnte in Obigem die Darstellungen des letzteren Autors in allen wesentlichen Puncten bestätigen und weiter ausführen, auch gelang es mir festzustellen, dass es die dritte Kiemenspalte ist, von der sich die Thymus ausstiilpt und. dass dieselbe nicht mit der seitlichen Thyreoidea-Anlage zusammenhängt.

Die Darstellung, die ich oben von der Entwicklung der Zunge gegeben habe, weicht von der, die in jüngster Zeit $\mathrm{Hi}$ s vertritt, in manchen Beziehungen ab. Ich verweise deswegen nochmals ausdrieklich auf die Figg. 6-9, die das von mir Beschriebene, glaube ich, in genügender Weise illustriren. Vor allen Dingen muss ich eine wesentliche Betheiligung des ventralen Endes der dritten Kiemenbogen am Aufbau des Organs in Abrede stellen; vielleicht hat man mitunter den in Fig. 7 sichtbaren Querwulst am Hinterrande meines Schaltstückes als zweiten Kiemenbogen geezählt und gelangte so dazu, dem dritten eine grössere Bedeutung 
zuzumessen. Ein Stadium, wie das von Embryo $\alpha$ von $\mathrm{His}$, bei dem die Anlage der Thyreoidea das vordere Ende einer Längsfurche darstellt, deren weitere Fortsetzung in den Kehlkopfschlitz und die Lungenanlagen führt, habe ich nicht gesehen. Die Schnittdicke von $1 / 10 \mathrm{~mm}$, die $\mathrm{His}$ benutzt hat, scheint mir für so kleine Objecte zu stark. His (I p. $\overline{\mathscr{S}} 4$ ) meint, dass das oberste (vorderste) Schlundbogenpaar sich an der Bildung der Zunge nicht oder höchstens mit seinem hinteren (unteren) Grenzabschnitte betheilige ; ich muss im Gegentheil constatiren, dass der ganze Körper der Zunge aus dem Schaltstiicke, das zwischen den Unterkieferfortsätzen der ersten Kiemenbogen und den nach hinten convergirenden Enden der zweiten gelegen ist, hervorwächst, während die zweiten Schlundbogen sammt dem sie verbindenden Theile des medianen Längskammes nur die Zungenwurzel liefern. Jenes Schaltstïck ist aber wohl eher zum ersten, als zum zweiten Kiemenbogen zu rechnen. Auch Dursy's und Kölliker's Schilderungen werden durch meinen Befund nicht ganz bestätigt. Dursy's Fig. 18 Taf. 1 stimmt auffâllig mit meiner Fig. 7, noch mehr aber der 'Text; denn D. bemerkt (Nr. XII) p. 121: „Der dem ersten Schlundbogen aufliegende Zungenkörper besteht aus zwei sich nach hinten verschmälernden Seitenwülsten, die durch ein breites Thal von einander geschieden werden. Im Grunde der letzteren bemerkt man eine keilförmig sich einschiebende breite Leiste, welche hinten in die Zungenwurzel tibergeht. Die beiden seitlichen Erhebungen und die Leiste dazwischen sind an meiner Fig. 7 ebenfalls zu sehen, sie stammen vom Schaltstücke, ausserdem gehören aber zu diesem die zunächst nach-hinten folgenden Querwülste, so dass sich mir sehr stark der Verdacht aufdrängt, D u rsy habe dieselben (mit dem Buchstaben c) fälschlich als zweite Kiemenbogen gezählt. Ist diese Vermuthung richtig, so blieben auch bei Dursy nur die zweiten Kiemenbogen mit ihrem Copularstiucke als Bildner der Zungenwurzel uibrig. Als Rest der dritten Kiemenbogen, die nach meinem Befunde sich nicht wesentlich am Aufbau der Zunge betheiligen, sondern im Wachsthum zurïckbleiben und später atrophiren, ist vielleicht in Dursy's Figur der kleine Querwulst auf der linken Seite am Hinterrande des zweiten (bei ihm dritten Kiemenbogens, Buchstabe d) zu deuten. Auffallend ist das geringe Längsmaass des Dursy'schen Rindsembryos 11,5 mm im Vergleich mit dem Fig. $7 \mathrm{zu}$ Grunde liegenden Schweinsembryo (13 N. L.). Am Besten stimmen meine Figuren 
mit denen der Reichert'schen Arbeit (X), die vor 45 Jahren erschienen ist, ibberein. Man vergleiche $\mathrm{X}$ Taf. VII Fig. 10-14 mit meinen Figg. 6-8; nur die Längsmaasse unserer Figuren sind etwas verschieden. Reichert beschreibt genau das Schaltstiick zwischen den ventralen Enden des ersten und zweiten Kiemenbogenpaares (1. c. p. 158) und weiss, dass dieses ,gleichschenklige Dreieck sich nach und nach in das Geschmacksorgan umwandelt" Reichert kennt dann die die ventralen Enden der Schlundbogen verbindende mediane Leiste (siehe Erklärung von Fig. 10 u. 11) und die Umbildung des hintern Endes derselben in die Epiglottis. Die Betheiligung der zweiten Kiemenbogen am Aufbau der Zunge bleibt bei Reichert im Unklaren, obgleich aus seinen Figuren die Bildung der Zungenwurzel aus denselben sich ziemlich deutlich erkennen lässt. Auch die Schicksale der ventralen Enden der dritten Kiemenbogen scheint $\mathrm{Reichert}$ nicht weiter verfolgt zu haben. - Die Epiglottis entsteht nach meiner Darstellung aus dem die dritten (und vielleicht auch die vierten) Kiemenbogen verbindenden Stücke der medianen Leiste, während die ventralen Enden der dritten Kiemenbogen selbst allmählich verschwinden.

Ein kurzes Resummé über die in dieser Arbeit gewonnenen Resultate ist in dem Referate des Vortrages enthalten, den ich am 1. December c. vor der medicinischen Section der schlesischen Gesellschaft für vaterländische Cultur gehalten habe; es liegt desshalb, glaube ich, kein Bedürfniss vor, an dieser Stellle ein solches zu wiederholen.

Breslau, im December 1882.

\section{Citirte Literatur.}

1. W. His, Anatomie menschlicher Embryonen. I. Embryonen des ersten Monats. Mit Atlas. Leipzig 1880.

2. - - II. Gestalt und Grössenentwicklung bis zum Schluss des zweiten Monats. Leipzig 1882.

3. - - Mittheilungen zur Embryologie der Säugethiere und des Menschen. Taf. XI und XII Archiv für Anatomie und Physiologie, anat. Abth. 1881.

4. L. Stieda, Untersuchungen über die Entwicklung der glandula thymus, glandula thyreoidea und glandula carotica. 2 Taf. Leipzig 1881.

drehiv f. mikrosk. Anatomie. Bd. 22. 
5. A. Wölfler, Ueber dic Entwicklung und den Bau der Schilddrüse. 7 Taf. und 4 Holzschnitte. Berlin 1881.

6. A. Ses sel, Zur Entwickelungsgeschichte des Vorderdarms. Taf. XX u. XXI Archiv für Anatomie und Physiologie, anat. Abth. 1877.

7. W. Moldenhauer, Die Entwicklung des mittleren und des äusseren Ohres. Taf. VI-IX und 2 Holzschnitte. Morphol. Jahrbuch III.

8. A. Kölliker, Entwickelungsgeschichte des Menschen und der höhern Thiere. 2. Aufl. Leipzig 1879.

9. F. M. Balfour, Handbuch der vergleichenden Embryologie, übersetzt von Vetter. B. II. 1881.

10. C. Reichert, Veber die Visceralbogen der Wirbeithiere im Allgemeinen und deren Metamorphose bei den Vögeln und Säugethieren. Archiv für Anatomie und Physiologie. 1837.

11. W. Mürller, Ueber die Entwicklung der Schilddrüse. Jena'sehe Zeitschrift B. VI, p. 428. 1871.

12. E. Dursy, Zur Entwickelungsgeschichte des Kopfes des Menschen und der höhern Wirbelthiere. Tübingen 1869.

\section{Erklärung der Tafeln $\mathrm{X}$ und XI.}

Alle Figuren sind nach Präparaten von Schweinsembryonen gezeichnet; es ist desshalb im Folgenden einfach Embryo = Schweinsembryo zu setzen.

Für alle Figuren gültige Bezeichnungen.

C. = Gehirn.

ch. = Chorda dorsalis.

Au. = Auge.

N. = Nase.

H. = Herz.

Hw. = Aeussere Wand der Pericardialhöhle.

P. = Pericardialhöhle.

$\mathrm{Hv} .=$ Herzvorhof.

Aw. = Aortenwurzel.

I-IV oder $\mathbf{A}_{1}-\mathbf{A}_{4}=$ erster bis vierter Aortenbogen.

G. = Gefäisse.

M. = Mundhöhle.

R. = Rachen.

La. = Kehlkopf.

Al. = Aditus laryngis. 
Tr. = Trachea.

Eg. = Epiglottis.

$\mathrm{Kb}_{1-4}=$ erster bis vierter Kiemenbogen.

Kx. = Schaltstïck zwischen dem ersten und zweiten Kiemenbogen.

$\mathrm{mL} .=$ mediane Leiste zwischen den ventralen Enden der zweiten bis vierten Kiemenbogen.

Z. = Zunge.

$\mathrm{Zk}$. = Zungenkörper.

$Z \mathrm{w} .=$ Zungenwurzel.

$\mathrm{iK}_{1-4}=$ innere erste bis vierte Kiemenfurche.

$\mathrm{eK}_{1-4}=$ äussere erste bis vierte Kiemenfurche.

$\mathrm{Ks}_{1-1}=$ erste bis vierte Kiementasche.

Th. = epitheliale Thymusanlage.

$\mathrm{mS} .=$ mediane oder vordere epitheliale Anlage der Thyreoidea.

IS. = laterale oder hintere epitheliale Anlage der Thyreoidea.

\section{Tafel X.}

Fig. 1. Kopfende eines Embryos von $7 \mathrm{~mm} \mathrm{~N}$. L. in der Seitenansicht. Derselbe ist vor dem Erhärten beim Loslösen des Amnion's spiralig gedreht worden, dabei wurde das Herz stark nach der in der Zeichnung vorliegenden Seite herausgedrängt. $x$ ein zwischen Kopf und Herz eingeklemmter Amnionfetzen. - conc. Pikrins.-Alk. - Vergrösserung 15 fach.

Fig. 2. Kopfende eines Embryos von $11 \mathrm{~mm}$ N. L. in der Seitenansicht. - Chromsäure-Alkohol. - Vergrösserung $15 f a c h$.

Fig. 3. Stück des Kopfendes von einem Embryo von $13 \mathrm{~mm}$ N. L. in der Seitenansicht. - Von 2 führt eine Linie ungefāhr $\mathrm{zu}$ dem Punkte am hintern Rande des zweiten Kiemenbogens, an dem das Ende der zweiten Kiemenspalte das Epithel der äussern Haut in der zweiten äussern Kiemenfurche erreicht (nach dem Modell eingezeichnet). Der Punkt unter dem Ende dieser Linie ist die Stelle, wo jetzt das Ende des dritten Kiemenspaltrestes mit der Epidermis verschmolzen ist. - Müller'sche Fl. - Alkohol. - Vergr. 15 fach.

Fig. 4. Stück eines Wachsmodells, das nach einer Schnittserie durch den Kopf eines Embryos von $7 \mathrm{~mm} \mathrm{~N}$. L. (aus derselben Tracht, wie der von Fig. 1) in 60 facher Vergrösserung gearbeitet ist. Es wurden die ganzen Schnitte auf die der Flächenvergrösserung entsprechend dicken Wachsplatten aufgezeichnet und dann Mundrachenhöhle und die Kiemenspalten so ausgeschnitten, als wenn denselben der Epithelüberzug fehite; dieselben erschienen also, nachdem die Platten mit- 
einander richtig verklebt waren, als relativ zu weite Räume. An den wichtigsten Stellen wurde die Verklebung nicht vollkommen ausgeführt, so dass eine Trennung des Modells in einzelne Stücke möglich blieb. So ist für die vorliegende Figur das Modell in einer Schnittebene $\left(2^{*}\right)$ anseinandergenommen, die den Hinterrand der zweiten innern Kiemenfurche trifft. - Man blickt von hinten und ein wenig von rechts auf die Schnittebene; nur die linke Hälfte des Modell-Stïckes ist dargestellt. Die Decke der Mundhöhle (mit Gehirn, Chorda u. s. w.) ist durch einen Schnitt, dessen Fläche in $1^{*}$ in der Verkürzung zu sehen ist, abgetrennt; ausserdem ist der eigentlich in das hintere (nicht dargestellte) Stück des Modells fallende hinterste Theil des zweiten Kiemenbogens durch einen schrägen Schuitt (Fläche $3^{*}$ ), der sich ventralwärts zwischen $\alpha$ und $\beta$ in die zweite äussere Kiemenfurche fortsetzt, abgenommen und auf das vorliegende Stück, um wenigstens die laterale Wand der Kiementasche vollständig zu machen, aufgeklebt. Denlkt man sich den zwischen den weissen punctirten Linien liegenden Streifen ausgefüllt; so wird damit die zweite innere Kiemenöffinung vollkommen begrenzt, man sieht, wie dieselbe von der Seitenwand der Mundhöhle aus tief in den Boden zwischen zweiten und dritten Kiemenbogen $\left(\mathrm{Kb}_{2}\right.$ und $\left.\mathrm{Kb}_{3}\right)$ bis nahe an die mediane Leiste (mL.) einschneidet. Unter $3 *$ führt der weisse Pfeil in die Fortsetzung der Kiementasche, die über das Niveau der Decke der Mundhöhle nach oben ausgebuchtet ist (vergl. auch Fig. 10). Zwischen $\boldsymbol{\alpha}$ und $\beta$ am hintern Rande der Kiementasche mündet dieselbe nach aussen $d$. h. revera verschmilzt ihr Epithel mit dem der zweiten äussern Kiemenfurche. An demselben Modell war auch die Herzwand (Hw.), die grossen Gefässe (Aw. u. II), sowie die Herzhöhlen ausgearbeitet, letztere sind aber nicht mehr in der Zeichnung enthalten. II deutet auf das vordere. Ende des ventralen Stammes der rudimentären zweiten Aortenbögen.

Fig. 5. Das sich an Figur 4 anschliessende Stück desselben Modells von $16 \mathrm{~mm}$ Dicke. Man blickt von hinten und ein wenig von links und oben auf die Schnittebene, die durch den Hinterrand der dritten Kiementasche $\left(\mathbf{K s}_{\mathbf{g}}\right)$ geht. Wieder ist der abgeschnittene Rest des dritten Kiemenbogens $\left(\mathrm{Kb}^{3}\right)$ von dem nächstfolgenden Stücke fortgenommen und auf das vorliegende aufgeklebt; der Wulst, auf den die Linie von $K_{3}$ weist, ragt also über die Schnittläche * gegen den Beschauer heraus. Zwischen $\alpha$ und $\beta$ findet die Ausmündung der dritten Kiementasche statt. $\gamma$ ist die Leiste auf der vordern Wand derselben, die den uach oben ausgebuchteten Raum abgrenzt. Der weisse Pfeil zeigt in die nach innen, vorn und ventralwärts gerichtete Ausstülpung der Kiementasche, die die Anlage der Thymus darstellt. IV deutet auf die schräg angeschnittenen vierten Aortenbogen, die innerhalb des vorliegenden Modellstückes sich miteinander 
und gleichzeitig mit der Ursprungsstelle der dritten Aortenbogen vereinigen.

Fig. 6. Boden der Mundhöhle von einem Embryo von $11 \mathrm{~mm} \mathrm{N.L.} \mathrm{(aus}$ derselben Tracht, wie Fig. 2) 15 mal vergrössert.

Fig. 7. Boden der Mundhöhle von einem Embryo von $13 \mathrm{~mm} \mathrm{~N}$. L. (aus derselben Tracht, wie der von Fig. 3), beinahe $15 \mathrm{mal}$ vergrössert.

Fig. 8. Boden der Mundhöhle von einem Embryo von 15 mm N. L. (16 S.S.); beinahe 15̄mal vergrössert.

Fig. 9. Boden der Mundhöhle von einem Embryo von $17 \mathrm{~mm} \mathrm{N.} \mathrm{L.} \mathrm{(19} \mathrm{S.} \mathrm{S);}$ beinahe 10mal vergrössert. Man sieht senkrecht auf die Zungenwurzel, in Folge dessen erblickt man das Vorderende der schon sehr gewölbten Zunge in starker Verkürzung.

\section{Tafel XI.}

Fig. 10. Schnitt durch den Kopf eines Fmbryos von $7 \mathrm{~mm}$ N.L. (wie Fig. 1, 4 und 5) mit der medianen vordern Anlage der Thyreoidea. Der Raum der zweiten Kiemenspalte $\mathrm{K}_{\mathbf{s}_{2}}$ war auf diesem Schnitte schon von der Mundhöhle M. abgetrennt; ich hielt es für zweckmässig, in der Figur die Verbindung beider nach dem zunächst vorhergehenden Schnitte wiederherstellen zu lassen. * Reste der auch auf diesem Schnitte noch getroffenen hintern Wand der zweiten innern Kiemenöffnung, man braucht sich die freien Spitzen beider Reste nur vereinigt zu denken und man erhält den Raum der zweiten Kiemenspalte wieder vollkommen abgeschlossen. o Blindsackartige Ausstülpung der zweiten Kiemenspalte dorsalwärts über die Decke der Mundhöhle hinaus. - Vergrösserung 50 fach.

Fig. 11. Schnitt in der Höhe der vorderen medianen Thyreoidea-Anlage von einem Embryo von $16 \mathrm{~mm}$ N. L. (18 mm S. S.). Die Balken des Epithelnetzes sind an manchen Stellen, z. B. am rechten Ende der $\mathrm{mS}$. etwas von der bindegewebigen Grundlage abgelöst. Der Thymusschlanch Th. ist an zwei Stellen getroffen. III Ventraler Theil des dritten Aortenbogens $=$ ea, Arteria carotis communis. $\mathrm{rv}=$ Anlage des Remus recurrens vagi. 70mal vergrössert.

Fig. 12. Aus derselben Serie 13 Schnitte $(1 / 50 \mathrm{~mm}$ Dicke) hinter den vorigen. Zwischen dem Ende der im vorigen Bilde dargestellten medianen Schilddrüsenanlage (mS.) und dem Anfang der in dieser Figur gezeichneten lateralen Schilddrüsen-Anlage liegen 6 Schnitte, in denen nichts von beiden Drüsenanlagen zu sehen ist. $\mathrm{Da}$ das ventrale Ende der kolbenförmigen, seitlichen Schilddrüsen-Anlage in diesem Schnitte nicht mehr enthalten war, wurde es aus den folgenden vier Schnitten ergänzt. rv. = Anlage der ramus recurrens vagi. - sd. Arteria subclavia dextra. - ap. = Stamm der Arteria pul- 
monalis. Links neben der Art. subcl. dextr. liegt ein kleines Gefäss, dessen Bedeutung ich nicht kenne. - Vergrösserung 70 fach.

Fig. 13. Rechte Hälfte der Schilddrüse eines Embryos von $21,5 \mathrm{~mm} \mathrm{~S}$. $\mathrm{S}$. laterale (IS.) und mediane (mS.) Anlage sind in der Verschmelzung begriffen. - Vergrösserung circa 80 fach.

Fig. 1t. Linke Hälfte der Schilddrüse eines Embryos von $21 \mathrm{~mm}$ S. S. (aus derselben Tracht, wie der von Fig. 13). Die einzelnen miteinander verschmelzenden Zellbalken der medianen und lateralen SchilddrïsenAnlage waren in diesem Präparate distincter, als in dem, das dem vorhergehenden Bilde zu Grunde lag. Das dorsale Ende der IS, mit dem deutlichen Lumen ist aus einem benachbarten Schnitte ergänzt.

\section{Ueber die Muskelspindeln nebst Bemerkungen über Structur, Neubildung und Innervation der quergestreiften Muskelfaser.}

Von

Dr. L. Bremer.

Hierzu Tafel XII und XIII.

In den fünfziger Jahren, bis zu Anfang der sechziger drehte sich der Streit über die Bildungsweise der quergestreiften Mnskelfaser vorzugsweise um den ein- oder mehrzelligen Ursprung derselben. Lebert, Remak und Köll iker vertraten den einzelligen Standpunkt, während zu der älteren Schwann'schen Lehre von den Spindelzellen, die sich mit den Spitzen aneinanderreihen und so verschmelzen sollten, Margo's Lehre von den "Sarcoplasten“ trat. Diese sollten in einem kernhaltigen Blasteme entstehen, sich dachziegelartig nebeneinander legen und so verschmelzend ein Primitivbündel bilden; und zwar sollte eine derartige Neubildung nicht 